\title{
Über heterogene Gleichgewichte von Metallhalogeniden mit Wasserstoff bzw. Chlorwasserstoff
}

Von der

Technischen Hochschule der Freien Stadt Danzig zur Erlangung der Würde eines Doktor-Ingenieurs

genehmigte

Dissertation

vorgelegt von

Dipl.-Ing. Reinhard Koop

Tag der Promotion: 12. November 1929

Referent: Prof. Dr. K. Jellinek

Korreferent: Prof. Dr. H. v. W artenberg

Akademische Verlagsgesellschaft m. b. H. Leipzig 1929 


\section{2}




\title{
Über heterogene Gleichgewichte von Metallhalogeniden mit Wasserstoff bzw. Chlorwasserstoff
}

\author{
Von der \\ Technischen Hochschule der Freien Stadt Danzig \\ zur Erlangung der Würde eines Doktor-Ingenieurs \\ genehmigte \\ Dissertation
}

vorgelegt von

Dipl.-Ing. Reinhard Koop

Tag der Promotion: 12. November 1929

Referent: Prof. Dr. K. Jellinek

Korreferent: Prof. Dr. H. v. Wartenberg

Akademische Verlagsgesellschaft m. b. H. Leipzig 1929 
Es werden Dampfspannungen von $\mathrm{FeCl}_{3}, \mathrm{CrCl}_{3}$ und $\mathrm{ZnCl}_{2}$ ermittelt. Es werden Reduktionsgleichgewichte von Metallhalogeniden mit $\mathrm{H}_{2}$ bestimmt und zwar von $\mathrm{CoF}_{3}$ zu $\mathrm{CoF}_{2}, \mathrm{MnF}_{2}, \mathrm{FeCl}_{3}$ zu $\mathrm{FeCl}_{2}, \mathrm{CrCl}_{3}$ zu $\mathrm{CrCl}_{2}, \mathrm{CrCl}_{2}, \mathrm{CuCl}_{2}$ zu $\mathrm{Cu}_{2} \mathrm{Cl}_{2}$. Es werden die Gleichgewichte von $\mathrm{CoF}_{2}, \mathrm{CdF}_{2}, \mathrm{ZnF}_{2}, \mathrm{MnF}_{2}$ und $\mathrm{NaF}$ mit $\mathrm{HCl}$-Gas gemessen.

\section{Reduktionsgleichgewichte von Metallfluoriden mit Wasserstoff.}

$$
\mathrm{HeF}_{\mathrm{a}}+\mathrm{H}_{\mathrm{2}} \rightleftarrows \mathrm{Me}+\boldsymbol{2} \mathrm{HF} \text {. }
$$

In den Arbeiten von JeLLINEK und RUdaT ${ }^{1}$ ) sind diese Gleichgewichte weitgehend gemessen worden. Hier sollen noch die Gleichgewichte von $\mathrm{CoF}_{3}$ und $\mathrm{MnF}_{2}$ mit Wasserstoff ermittelt werden.

An der Apparatur wurde im wesentlichen nichts geändert. Der in einem KrPPschen Apparat entwickelte Wasserstoff geht durch mehrere Pufferflaschen und eine Flasche mit alkalischer Permanganatlösung, sodann strömt das Gas durch einen Druckregulator und ein Kapometer und wird weiterhin in einem mit Pt-Asbest gefüllten und erhitzten Porzellanrohr von eventuell mitgeführtem Sauerstoff befreit. Vor Eintritt in das Reaktionsrohr wird der Wasserstoff in einem $\mathrm{CaCl}_{2}$-Turm und einem Rohr mit $\mathrm{P}_{2} \mathrm{O}_{5}$ getrocknet.

Der Ofen ist ein mit Chromnickeldraht bewickeltes Porzellanrohr (600 $\mathrm{mm}$ lang, $30 \mathrm{~mm}$ weit), das in Magnesia eingebettet ist. Das zur Temperaturmessung benutzte Thermoelement war bei $800^{\circ}$ (Schmelzpunkt von $\mathrm{NaCl}$ ) geeicht und befand sich in einem engen Eisenrohr. Da die Gleichgewichtsmessungen zum Teil bei hoher Temperatur vorgenommen wurden, musste ein Reaktionsrohr aus Nickel $(850 \mathrm{~mm}$ lang, $13 \mathrm{~mm}$ weit) gewählt werden. Die Temperatur in beiden Rohren, die an ihren Enden mit Bleikühlschlangen umwickelt waren, blieb über eine mittlere Strecke von $15 \mathrm{~cm}$ konstant. Wie vergleichende Messungen mit zwei Thermoelementen ergaben, war die Temperatur im Thermoelementschutzrohr um $5^{\circ}$ höher als im Reaktionsrohr.

1) JeLlinek und Rudat, Z. anorgan. u. allgem. Chem. 175, 281. 1928. 
Dieser Unterschied ist bei den endgültigen Temperaturangaben berücksichtigt worden.

Die Arbeitsweise ist dieselbe geblieben wie früher. Der gereinigte und getrocknete Wasserstoff wird mit konstanter Strömungsgeschwindigkeit über das Metallfluorid im Nickelschiff geleitet. Das entweichende Gasgemisch $\left(\mathrm{HF}+\mathrm{H}_{2}\right)$ wird analysiert, der Fluorwasserstoff in einer paraffinierten Vorlage mit $1 /{ }_{10}$ norm. $\mathrm{NaOH}$ absorbiert, der Wasserstoff in einem dahintergeschalteten Messkolben über Wasser aufgefangen. Ist der Messkolben bis zur Marke mit $H_{2}$ gefüllt, dann wird der Versuch abgestellt und der Inhalt der Vorlage mit 1/10 norm. $\mathrm{HCl}$ und Phenolphthalein in einer Platinschale bei Siedehitze titriert.

Bei allen Reduktionsgleichgewichten waren zwei feste Phasen mit konstantem Dampfdruck vorhanden. Nach dem Massenwirkungsgesetz gilt für die Reaktion $\mathrm{MeF}_{2}+\mathrm{H}_{2} \rightleftarrows \mathrm{Me}+2 \mathrm{HF}$ :

$$
\frac{p_{H F}^{2} \cdot p_{M e}}{p_{H_{2}} \cdot p_{M e F_{2}}}=K \quad \text { bzw. } \quad \frac{p_{H F}^{2}}{p_{H_{2}}}=K^{\prime} .
$$

Nach der Phasenregel ist bei drei Bestandteilen und drei Phasen die Anzahl der Freiheiten 2. Wenn über $T$ und $p$ (1 Atm.) verfügt ist, liegt im Gleichgewicht die Zusammensetzung der Gasphase fest.

In den folgenden Tabellen ist für jedes Metallfluorid eingetragen: die absolute Versuchstemperatur $T$, die aufgefangenen und auf $0^{\circ}$ und 1 Atm. reduzierten Kubikzentimeter $H_{2}$, die durch Titration ermittelten Kubikzentimeter $H F\left(0^{\circ}, 1 \mathrm{Atm}\right.$.), ferner die Strömungsgeschwindigkeit, d. h. der Quotient aus den Kubikzentimetern $H_{2}+1 / 2 \mathrm{~cm}^{3} H F$ und der Versuchsdauer in Minuten ( $\mathrm{cm}^{3} / \mathrm{Min}$.), und schliesslich die Volumprozente $H F$ (Volumproz. HF). Durch Extrapolation werden die Volumprozente $H F$ bei der Strömungsgeschwindigkeit Null ermittelt, wie dies früher am Beispiel des $\mathrm{PbF}_{2}$ gezeigt wuirde. Betreffs der Genauigkeit der Daten sei auf die frühere Arbeit von JELLINEK und Rudat verwiesen.

Das verwendete $\mathrm{CoF}_{3}$ war in liebenswürdiger Weise von Herrn Prof. RufF, Breslau, zur Verfügung gestellt worden, wofür an dieser Stelle bestens gedankt sei. Zwecks Analyse wurde das gelbbraune Präparat ${ }^{1}$ ) mit wenig überschüssiger $\mathrm{H}_{2} \mathrm{SO}_{4}$ in einer Platinschale eingedampft und als Sulfat bestimmt ${ }^{2}$ ). Die Analyse ergab $50 \cdot 84 \% \mathrm{Co}$, während die Formel $50 \cdot 83 \%$ Co verlangt. Das zurückbleibende Reduktionsprodukt obiger Reaktion war rosafarbiges $\mathrm{CoF}_{2}$.

1) RufF, Z. angew. Chem. 41, 737. 1928. Analytische Chemie, 11. Aufl., Bd. II, S. 116.

2) Näheres siehe Treadwell, 


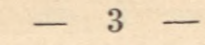

Tabelle 1. $2 \mathrm{CoF}_{3}+\mathrm{H}_{2} \rightleftarrows 2 \mathrm{CoF}_{2}+2 \mathrm{HF}$.

\begin{tabular}{|c|c|c|c|c|}
\hline$T$ & $\mathrm{em}^{3} H_{2}$ & $\mathrm{~cm}^{3} H F$ & $\mathrm{~cm}^{3} / \mathrm{Min}$ & $\begin{array}{c}\text { Volumproz. } \\
H F\end{array}$ \\
\hline 470 & $\begin{array}{c}231 \cdot 23 \\
231 \cdot 23 \\
231 \cdot 23 \\
-\end{array}$ & $\begin{array}{l}0.97 \\
1.58 \\
2.58 \\
-\end{array}$ & $\begin{array}{l}6 \cdot 30 \\
4 \cdot 50 \\
1 \cdot 88 \\
0\end{array}$ & $\begin{array}{l}0.42 \\
0.68 \\
1.10 \\
1.27\end{array}$ \\
\hline 527 & $\begin{array}{c}91.91 \\
91.91 \\
91.91 \\
-\end{array}$ & $\begin{array}{c}3.48 \\
4 \cdot 88 \\
6 \cdot 13 \\
-\end{array}$ & $\begin{array}{l}8 \cdot 75 \\
\tilde{5} \cdot 02 \\
1.55 \\
0\end{array}$ & $\begin{array}{l}3 \cdot 6 \tilde{5} \\
5 \cdot 04 \\
6 \cdot 25 \\
6 \cdot 81\end{array}$ \\
\hline 595 & $\begin{array}{c}93.01 \\
93 \cdot 01 \\
93.01 \\
-\end{array}$ & $\begin{array}{c}41 \cdot 67 \\
43 \cdot 36 \\
46 \cdot 24 \\
-\end{array}$ & $\begin{array}{l}8 \cdot 13 \\
5 \cdot 61 \\
1 \cdot 60 \\
0\end{array}$ & $\begin{array}{l}30 \cdot 93 \\
31 \cdot 80 \\
33 \cdot 21 \\
33 \cdot 70\end{array}$ \\
\hline
\end{tabular}

Tabelle 2. $M n F_{2}+H_{2} \rightleftarrows M n+2 H F$.

\begin{tabular}{|c|c|c|c|c|}
\hline$T$ & $\mathrm{~cm}^{3} H_{2}$ & $\mathrm{~cm}^{3} H F$ & $\mathrm{~cm}^{3} / \mathrm{Min}$. & $\begin{array}{c}\text { Volumproz. } \\
H F\end{array}$ \\
\hline 1175 & $\begin{array}{c}230 \cdot 6 \\
230 \cdot 6 \\
230 \cdot 9 \\
-\end{array}$ & $\begin{array}{c}2.003 \\
3.12 \\
3.43 \\
-\end{array}$ & $\begin{array}{l}8.15 \\
5 \cdot 80 \\
4.01 \\
0\end{array}$ & $\begin{array}{l}1.08 \\
1.33 \\
1.46 \\
1.8 \tilde{5}\end{array}$ \\
\hline 1273 & $\begin{array}{c}233 \cdot 5 \\
230 \cdot 8 \\
232.9 \\
-\end{array}$ & $\begin{array}{c}4 \cdot 18 \\
4 \cdot 97 \\
5 \cdot 72 \\
-\end{array}$ & $\begin{array}{l}9.06 \\
5 \cdot 83 \\
2 \cdot 48 \\
0\end{array}$ & $\begin{array}{l}1.75 \\
2 \cdot 10 \\
2.39 \\
2.60\end{array}$ \\
\hline 1376 & $\begin{array}{c}228.7 \\
228.7 \\
228.7 \\
-\end{array}$ & $\begin{array}{c}12 \cdot 99 \\
14 \cdot 32 \\
15 \cdot 36 \\
-\end{array}$ & $\begin{array}{l}7 \cdot 01 \\
4 \cdot 33 \\
1.99 \\
0\end{array}$ & $\begin{array}{l}\tilde{5} \cdot 38 \\
5 \cdot 89 \\
6 \cdot 29 \\
6 \cdot 67\end{array}$ \\
\hline
\end{tabular}

Das verwendete $\mathrm{MnF}_{2}$ war ein Kahlbaumsches Präparat. Es wurde im $H F$-Strom getrocknet und analysiert. Die Analyse ergab $58.6 \% \mathrm{Mn}$ als $\mathrm{MnSO}_{4}$ bestimmt, während die Formel $59 \cdot 1 \% \mathrm{Mn}$ verlangt. Das zurückbleibende Reduktionsprodukt war feinverteiltes Mn-Metall.

Berechnung der Fluortensionen und Bildungswärmen der Metallfluoride.

Wir bilden für jedes Metallfluorid den Ausdruck $\log \frac{p_{H F}^{2}}{p_{H_{2}}}$, wo die Partialdrucke in Atmosphären einzusetzen sind. Ebenso berechnen wir für dieselbe Temperatur den Ausdruck der HF-Dissoziation ${ }^{1}$ )

1) Zur Ableitung dieser Formel vgl. JellineK und Rudat, Z. anorgan. u. allgem. Chem. 175, 300. 1928. Wie wir nach Niederschrift der Arbeit fanden, hat O. Тн. Krefft in einer unter Leitung von Fredenhagen durchgeführten Dịser- 


$$
\begin{gathered}
-4- \\
\log \frac{p_{H F}^{2}}{p_{H_{2}} \cdot p_{F_{2}}}=\frac{28000}{T}+1 \cdot 05 .
\end{gathered}
$$

Aus der Gleichung:

$$
\log \frac{p_{H F}^{2}}{p_{H_{2}} \cdot p_{F_{2}}}-\log \frac{p_{H F}^{2}}{p_{H_{2}}}=-\log p_{F_{2}},
$$

wo der zweite Ausdruck links sich auf die Reduktionsgleichgewichte bezieht (siehe Fig. 1), ergibt sich dann $-\log p_{F_{2}}$, wo auch die Fluortension $p_{F_{2}}$ in Atmosphären gemessen ist.

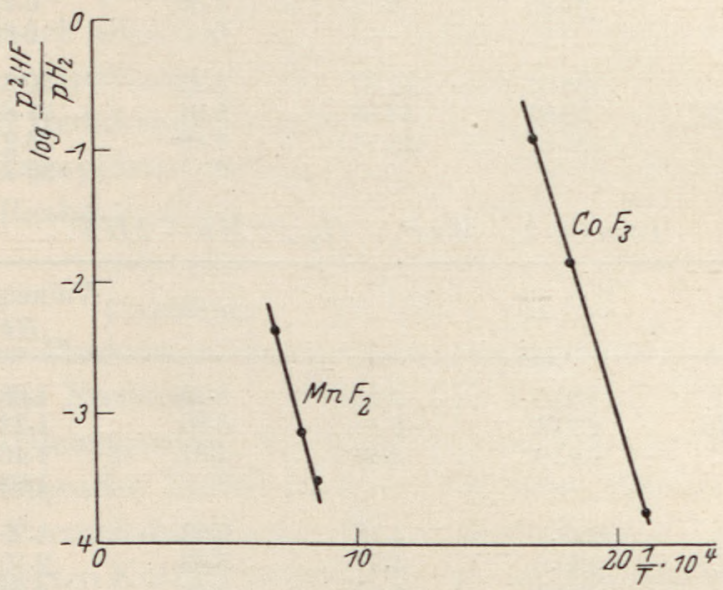

Fig. 1 .

tation „,Die elektrolytische Darstellung des Fluor. Messung der Wasserstoff-Fluorknallgaskette und Untersuchungen über die Messung der Alkali-Säurekette in Flusssäure", Greifswald 1928, durch direkte Messung der EMK der $H_{\varepsilon}, F_{2}$-Kette den Wert $2 \cdot 768$ Volt bei $T=273$ und als Polarisationsspannung in einer Kaliumbifluoridschmelze den Wert $2 \cdot 8$ Volt bei $T=523$ gefunden. Nach der Formel:

$$
\frac{2 \cdot 96500 \cdot E}{4 \cdot 188}=4 \cdot 571 \cdot T\left(\log \frac{p_{H_{2}} p_{F_{2}}}{p_{H F}^{2}}-\log K_{p}\right)
$$

ergeben sich dann für $p_{\mathrm{H}_{2}}=1 \mathrm{Atm}$. und $p_{\mathrm{F}_{2}}=1 \mathrm{Atm}$. die folgenden Werte, die mit den aus der von JELLINEK aufgestellten Formel folgenden verglichen sind:

\begin{tabular}{c|c|c|c|c|c}
\hline$T$ & $\begin{array}{c}p_{H F} \\
\text { Atm. }\end{array}$ & $\begin{array}{c}\text { EMK } \\
\text { Volt }\end{array}$ & $\begin{array}{c}\log K_{p} \\
\text { FredeN- } \\
\text { HAGEN }\end{array}$ & $\begin{array}{c}\log K_{p} \\
\text { JeLLINEK }\end{array}$ & $\begin{array}{c}\text { Differenz } \\
\text { JELLINEK- } \\
\text { FrEDENHAGEN }\end{array}$ \\
\hline 273 & 0.467 & 2.768 & 101.79 & 103.65 & $1.8 \%$ \\
523 & 0.092 & 2.8 & 52.08 & 54.60 & $5.0 \%$
\end{tabular}

Die Differenz der beiden $K_{p}$-Werte dürfte innerhalb der Fehlergrenze der Messungen liegen. 
In der Tabelle 3 sind für die beiden Metallfluoride die Werte $\log \frac{p_{H F}^{2}}{p_{H_{2}} \cdot p_{F_{2}}}$ ferner $\log \frac{p_{H F}^{2}}{p_{H_{2}}}$ und die dazugehörigen Werte $\log p_{F_{2}}$ eingetragen. Tabelle 3 .

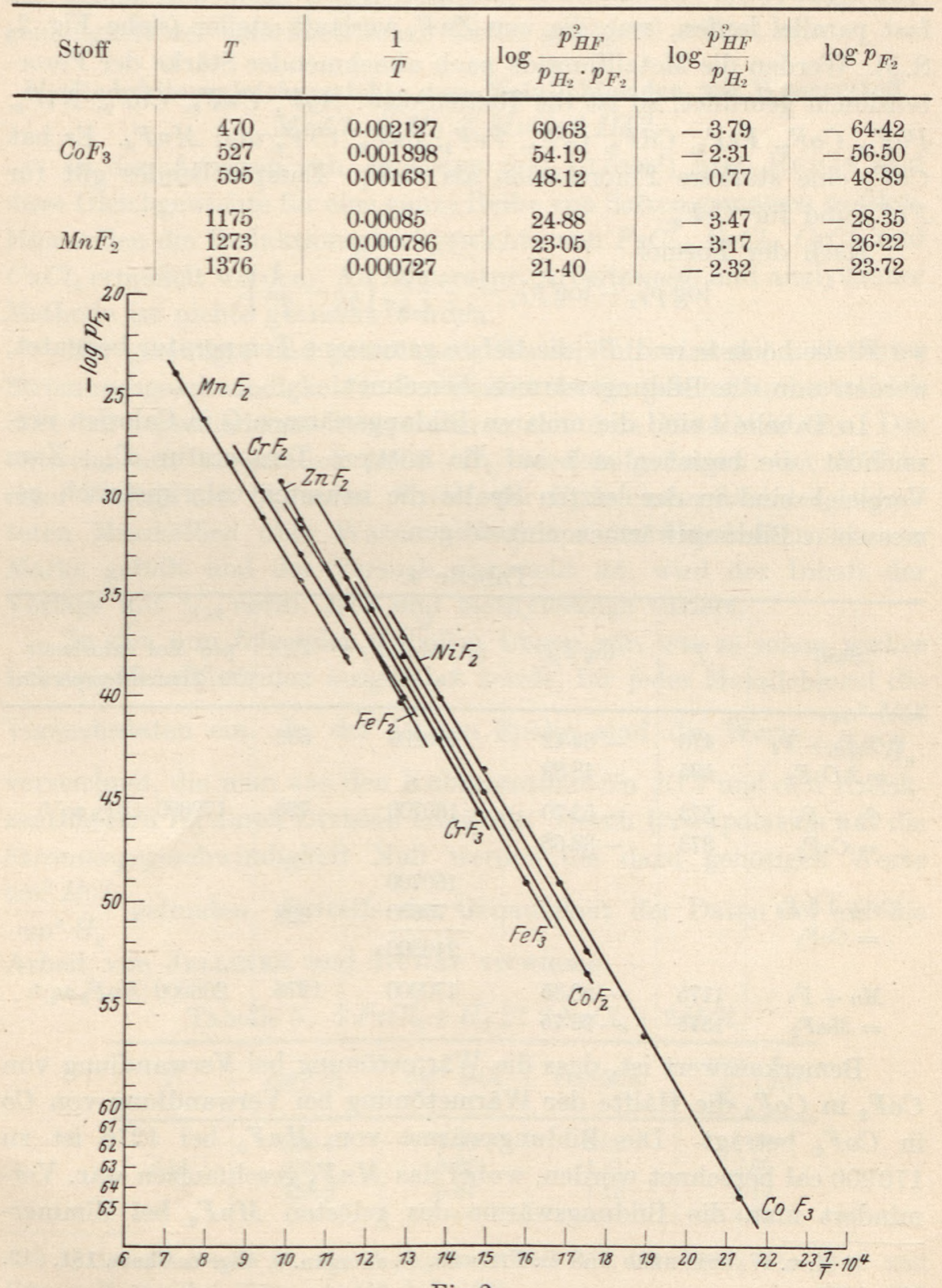

Fig. 2. 
Werden für die Metallfluoride der Tabelle 3 und für einige verwandte Fluoride aus der Arbeit von JELLINEK und RUdat die $\log p_{F_{2}}$ gegen $\frac{1}{T} \cdot 10^{4}$ aufgetragen, so ergeben sich gerade Linien, die einander fast parallel laufen, nur die von $Z n F_{2}$ verläuft steiler (siehe Fig. 2, S. 5). Werden die Metallfluoride nach abnehmender Stärke der Fluortensionen geordnet, so ist die Reihenfolge: $\mathrm{AgF}, \mathrm{CuF}_{2}, \mathrm{CoF}_{3}, \mathrm{NiF}_{2}$, $\mathrm{PbF}_{2}, \mathrm{CoF}_{2}, \mathrm{FeF}_{3}, \mathrm{CdF}_{2}, \mathrm{CrF}_{3}, \mathrm{FeF}_{2}, \mathrm{ZnF}_{2}, \mathrm{CrF}_{2}$ und $\mathrm{MnF}_{2}$. Es hat $\mathrm{CoF}_{3}$ eine stärkere Fluortension als $\mathrm{CoF}_{2}$. Entsprechendes gilt für $\mathrm{FeF}_{3}$ und für $\mathrm{CrF}_{3}$.

Nach der Formel

$$
\log p_{F_{2}}^{\prime}-\log p_{F_{2}}^{\prime \prime}=-\frac{Q}{4 \cdot 57}\left(\frac{1}{T^{\prime}}-\frac{1}{T^{\prime \prime}}\right),
$$

wo $T^{\prime}$ die höchste und $T^{\prime \prime}$ die tiefste gemessene Temperatur bedeutet, werden nun die Bildungswärmen berechnet.

In Tabelle 4 sind die molaren Bildungswärmen $Q$ in Calorien verzeichnet; sie beziehen sich auf die mittlere Temperatur $T_{m}$. Zum Vergleich sind in der letzten Spalte die neuesten calorimetrisch gemessenen Bildungswärmen eingetragen.

Tabelle 4.

\begin{tabular}{|c|c|c|c|c|c|}
\hline Stoff & $T$ & $\log p_{F_{2}}$ & $\begin{array}{l}Q \\
\text { pro Mol } F . y \\
\text { in eal }\end{array}$ & $T_{m}$ & $\begin{array}{l}Q \\
\text { pro Mol calorimetr. } \\
\text { für Zimmertemperatur }\end{array}$ \\
\hline $\begin{array}{c}2 \mathrm{CoF}_{2}+\mathrm{F}_{2} \\
=2 \mathrm{CoF}_{3}\end{array}$ & $\begin{array}{l}470 \\
595\end{array}$ & $\begin{array}{l}-64.42 \\
-48 \cdot 89\end{array}$ & 159200 & 533 & \\
\hline $\begin{array}{l}C o+F_{2} \\
=C_{o} F_{2}\end{array}$ & $\begin{array}{l}573 \\
873\end{array}$ & $\begin{array}{l}-53 \cdot 70 \\
-32 \cdot 68\end{array}$ & 160700 & 723 & $172800\left(C o F_{2} \mathrm{aq}^{\prime 1}\right)$ \\
\hline $\begin{array}{c}C o+1.5 F_{2} \\
=C_{o} F_{3}\end{array}$ & & & $\begin{array}{r}160700 \\
+\quad 79600 \\
240300\end{array}$ & 628 & \\
\hline $\begin{array}{l}M n+F_{2} \\
=M n F_{2}\end{array}$ & $\begin{array}{l}1175 \\
1376\end{array}$ & $\begin{array}{l}-28.35 \\
-23.75\end{array}$ & 170900 & 1276 & $\left.206000\left(M n F_{2} \mathrm{aq}\right)^{1}\right)$ \\
\hline
\end{tabular}

Bemerkenswert ist, dass die Wärmetönung bei Verwandlung von $\mathrm{CoF}_{2}$ in $\mathrm{CoF}_{3}$ die Hälfte der Wärmetönung bei Verwandlung von $\mathrm{Co}$ in $\mathrm{CoF}_{2}$ beträgt. Die Bildungswärme von $\mathrm{MnF}_{2}$ bei 1276 ist zu 170900 cal berechnet worden, wobei das $M n F_{2}$ geschmolzen war. Vermindert man die Bildungswärme des gelösten $\mathrm{MnF}_{2}$ bei Zimmer-

1) H. v. WARtenberg und O. Fitzner, Z. anorgan. u. allgem. Chem. 151, 313, 330. 1926. 
temperatur, das ist $206000 \mathrm{cal}$, um die mutmassliche Lösungswärme, das ist $15000 \mathrm{cal}$, so erhält man $191000 \mathrm{cal}$. Die Differenz von $10 \%$ zwischen 191000 und $171000 \mathrm{cal}$ dürfte durch die Temperaturdifferenz, auf welche sich beide Daten beziehen, sowie durch eine etwaige Ungenauigkeit der calorimetrischen Daten genügend erklärt sein.

\section{Reduktionsgleichgewichte von Metallchloriden mit Wasserstoff. $\mathrm{MeCl}_{2}+\mathrm{H}_{2} \rightleftarrows \mathrm{Me}+2 \mathrm{HCl}$.}

In den Arbeiten von Jellinek und Uloth ${ }^{1}$ ) bzw. Rudat sind diese Gleichgewichte für eine ganze Reihe von Salzen gemessen worden. Hier sollen die Reduktionsgleichgewichte von $\mathrm{FeCl}_{3}, \mathrm{CrCl}_{3}, \mathrm{CrCl}_{2}$ und $\mathrm{CuCl}_{2}$ ermittelt werden. An Apparatur, Arbeitsweise und analytischer Methode ist nichts geändert worden.

Der gereinigte und getrocknete Wasserstoff wird mit konstanter Strömungsgeschwindigkeit in das Reaktionsrohr geleitet und setzt sich dort bei verschiedenen Temperaturen mit dem Metallsalz um. Das entweichende Gasgemisch $\left(\mathrm{HCl}+\mathrm{H}_{2}\right)$ wird aufgefangen: der $\mathrm{HCl}$ in einer Vorlage mit $1 / 10$ norm. $\mathrm{NaOH}$, der $\mathrm{H}_{2}$ in einem dahintergeschalteten Messkolben über Wasser. Nachdem der Messkolben bis zur Marke gefüllt und der Versuch abgestellt ist, wird der Inhalt der Vorlage mit 1/10 norm. $\mathrm{HCl}$ und Methylorange titriert.

In den nun folgenden Tabellen tragen wir, wie es schon vorher bei den Metallfluoriden ausgeführt wurde, für jedes Metallchlorid die Versuchsdaten ein. In der letzten Spalte sind die Werte $\frac{\mathrm{cm}^{3} \mathrm{HCl}}{\mathrm{cm}^{3} \mathrm{H}_{2}}$ verzeichnet, die man aus den Kubikzentimetern $\mathrm{HCl}$ und den Kubikzentimetern $\mathrm{H}_{2}$ durch Division ermittelt. Durch Extrapolation auf die Strömungsgeschwindigkeit Null werden die dazu gehörigen Werte $\frac{\mathrm{cm}^{3} \mathrm{HCl}}{\mathrm{cm}^{3} \mathrm{H}_{2}}$ gefunden. Betreffs der Genauigkeit der Daten sei auf die Arbeit von JELLINEK und Rudat verwiesen.

\begin{tabular}{c|c|c|c|c}
\multicolumn{4}{c}{ Tabelle 5. $2 \mathrm{FeCl}_{3}+\mathrm{H}_{2} \rightleftarrows 2 \mathrm{FeCl}_{2}+2 \mathrm{HCl}}$. \\
\hline \multirow{2}{*}{$T$} & $\mathrm{~cm}^{3} \mathrm{H}_{2}$ & $\mathrm{~cm}^{3} \mathrm{HCl}$ & $\mathrm{cm}^{3} / \mathrm{Min}$. & $\frac{\mathrm{HCl}}{\mathrm{H}_{2}} \cdot 10^{2}$ \\
\hline \multirow{3}{*}{546} & $232 \cdot 1$ & $4 \cdot 60$ & $8 \cdot 08$ & $1 \cdot 98$ \\
& $232 \cdot 1$ & $8 \cdot 82$ & $4 \cdot 31$ & $3 \cdot 80$ \\
& $139 \cdot 3$ & $5 \cdot 81$ & $1 \cdot 16$ & $4 \cdot 17$ \\
& - & - & 0 & $4 \cdot 20$
\end{tabular}

1) Jellinek und Uloth, Z. physikal. Chem. 119, 161. 1926. Jellinek und Rudat, Z. physikal. Chem. (A) 143, 244. 1929. 
Das wasserfreie, schwarze, metallisch glänzende Salz wurde durch Sublimation eines reinen Kahlbaumschen Präparats nochmals gereinigt. Die Messungen konnten nicht zu höheren Temperaturen fortgesetzt werden, da das $\mathrm{FeCl}_{3}$ bei etwas höherer Temperatur bereits zu stark sublimierte. Das durch Reduktion erhaltene $\mathrm{FeCl}_{2}$ war gelblichweiss.

\begin{tabular}{|c|c|c|c|c|}
\hline$T$ & $\mathrm{~cm}^{3} \mathrm{H}_{2}$ & $\mathrm{~cm}^{3} \mathrm{HCl}$ & $\mathrm{cm}^{3} / \mathrm{Min}$. & $\frac{\mathrm{HCl}}{\mathrm{H}_{\mathrm{Q}}}$ \\
\hline 689 & $\begin{array}{c}93 \cdot 42 \\
93 \cdot 42 \\
93 \cdot 42 \\
-\end{array}$ & $\begin{array}{r}20.65 \\
103.70 \\
144.72 \\
-\end{array}$ & $\begin{array}{r}10 \cdot 02 \\
6 \cdot 65 \\
2.93 \\
0\end{array}$ & $\begin{array}{l}0.22 \\
1.11 \\
1.55 \\
1.67\end{array}$ \\
\hline 718 & $\begin{array}{c}46 \cdot 52 \\
46 \cdot 52 \\
46 \cdot \tilde{2} 2 \\
-\end{array}$ & $\begin{array}{r}79.02 \\
130.23 \\
154.13 \\
-\end{array}$ & $\begin{array}{l}7 \cdot 12 \\
4 \cdot 65 \\
2 \cdot 29 \\
0\end{array}$ & $\begin{array}{l}1.70 \\
2.79 \\
3.31 \\
3.48\end{array}$ \\
\hline 783 & $\begin{array}{c}46 \cdot 13 \\
46 \cdot 13 \\
46 \cdot 13 \\
-\end{array}$ & $\begin{array}{c}237 \cdot 10 \\
292.93 \\
323 \cdot 31 \\
-\end{array}$ & $\begin{array}{l}9 \cdot 33 \\
5 \cdot 67 \\
2 \cdot 48 \\
0\end{array}$ & $\begin{array}{l}5 \cdot 14 \\
6 \cdot 35 \\
7.01 \\
7 \cdot 31\end{array}$ \\
\hline
\end{tabular}

Das $\mathrm{CrCl}_{3}$ war ein reines Kahlbaumsches Präparat von violetter Farbe. Das Reduktionsprodukt war grauweisses $\mathrm{CrCl}_{2}$.

Tabelle 7. $\mathrm{CrCl}_{2}+\mathrm{H}_{2} \rightleftarrows \mathrm{Cr}+2 \mathrm{HCl}$.

\begin{tabular}{|c|c|c|c|c|}
\hline$T$ & $\mathrm{~cm}^{3} \quad H_{2}$ & $\mathrm{em}^{3} \mathrm{HCl}$ & $\mathrm{cm}^{2} / \mathrm{Min}$ & $\frac{\mathrm{HCl}}{\mathrm{H}_{2}}$ \\
\hline 1294 & $\begin{array}{c}93 \cdot \tilde{0} 0 \\
93 \cdot 50 \\
92 \cdot 87 \\
-\end{array}$ & $\begin{array}{r}9 \cdot 89 \\
9 \cdot 82 \\
10 \cdot \text { อ̃ } \\
-\end{array}$ & $\begin{array}{l}6 \cdot 1 \tilde{5} \\
4 \cdot 10 \\
1.38 \\
0\end{array}$ & $\begin{array}{l}0.106 \\
0.105 \\
0.114 \\
0 \cdot 114\end{array}$ \\
\hline 1408 & $\begin{array}{c}93.01 \\
93.01 \\
93.01 \\
-\end{array}$ & $\begin{array}{c}16 \cdot 93 \\
17 \cdot 43 \\
17.18 \\
-\end{array}$ & $\begin{array}{l}6 \cdot 34 \\
5 \cdot 98 \\
2 \cdot 12 \\
0\end{array}$ & $\begin{array}{l}0.182 \\
0.187 \\
0.18 j \\
0.185\end{array}$ \\
\hline 1465 & $\begin{array}{c}92.87 \\
92.87 \\
92.87 \\
-\end{array}$ & $\begin{array}{c}19.71 \\
20.00 \\
22.09 \\
-\end{array}$ & $\begin{array}{l}5 \cdot 14 \\
4 \cdot 67 \\
2 \cdot 26 \\
0\end{array}$ & $\begin{array}{l}0.212 \\
0.215 \\
0.238 \\
0.24 \tilde{5}\end{array}$ \\
\hline
\end{tabular}

Das $\mathrm{CrCl}_{2}$ wurde durch Reduktion von $\mathrm{CrCl}_{3}$ erhalten, seine Farbe war grauweiss. Das durch die Reduktion erhaltene $C r$-Metall war in feinen Nadeln kristallisiert. 
Tabelle 8. $2 \mathrm{CuCl}_{2}+\mathrm{H}_{2} \rightleftarrows \mathrm{Cu}_{2} \mathrm{Cl}_{2}+2 \mathrm{HCl}$.

\begin{tabular}{|c|c|c|c|c|}
\hline$T$ & $\mathrm{~cm}^{3} \quad H_{2}$ & $\mathrm{~cm}^{3} \mathrm{HCl}$ & $\mathrm{cm}^{3} /$ Min. & $\frac{\mathrm{HCl}}{\mathrm{H}_{2}}$ \\
\hline 524 & $\begin{array}{c}46 \cdot 76 \\
46 \cdot 76 \\
46 \cdot 76 \\
-\end{array}$ & $\begin{array}{c}10 \cdot 38 \\
17 \cdot 33 \\
24 \cdot 79 \\
-\end{array}$ & $\begin{array}{l}5 \cdot 19 \\
3 \cdot 46 \\
1 \cdot 8 \tilde{5} \\
0\end{array}$ & $\begin{array}{l}0.222 \\
0.467 \\
0.530 \\
0.57\end{array}$ \\
\hline 598 & $\begin{array}{c}46 \cdot 90 \\
46 \cdot 90 \\
46 \cdot 90 \\
-\end{array}$ & $\begin{array}{c}43 \cdot 32 \\
59 \cdot 14 \\
97 \cdot 92 \\
-\end{array}$ & $\begin{array}{l}6 \cdot 23 \\
4 \cdot 78 \\
1.47 \\
0\end{array}$ & $\begin{array}{l}0.924 \\
1.261 \\
2.087 \\
2.30\end{array}$ \\
\hline 670 & $\begin{array}{c}46.40 \\
46 \cdot 40 \\
46 \cdot 40 \\
-\end{array}$ & $\begin{array}{c}215 \cdot 91 \\
231 \cdot 50 \\
251 \cdot 02 \\
-\end{array}$ & $\begin{array}{l}5 \cdot 93 \\
4 \cdot 49 \\
2-12 \\
0\end{array}$ & $\begin{array}{l}4.65 \\
4.99 \\
5.41 \\
5.58\end{array}$ \\
\hline
\end{tabular}

Das $\mathrm{CuCl}_{2}$ war ein reines Kahlbaumsches Präparat von brauner Farbe. Das durch Reduktion erhaltene $\mathrm{Cu}_{2} \mathrm{Cl}_{2}$ war durch Beimengung von zum Teil noch nicht reduziertem $\mathrm{CuCl}_{2}$ gelb-hellbraun gefärbt.

Berechnung der Tensionen des zweiatomigen Chlors und der Bildungswärmen der Chloride.

Wir bilden zunächst für jedes Metallchlorid den Ausdruck

$$
\frac{1}{K_{1}}=\frac{p_{H C l}^{2}}{p_{H_{2}}},
$$

sodann den Ausdruck für die $\mathrm{HCl}$-Dissoziation:

$$
\left.\log K_{2}=\log \frac{p_{\mathrm{H}_{2}} \cdot p_{\mathrm{Cl}_{2}}}{p_{\mathrm{HCl}}^{2}}=-\frac{9554}{T}+0 \cdot 553 \log T-2 \cdot 42^{1}\right) .
$$

Aus der Formel

$$
\log p_{C_{2}}=\log \left(K_{2} \cdot \frac{1}{K_{1}}\right)
$$

ergibt sich $\log p_{\mathrm{Cl}_{2}}$, wo auch die $\mathrm{Cl}_{2}$-Tension in Atmosphären gemessen ist. Wir können bei den meisten untersuchten Chloriden mit genügender Genauigkeit setzen

$$
p_{\mathrm{HCl}}+p_{\mathrm{H}_{2}}=1 \text {. }
$$

Bezeichnen wir dann $\frac{p_{H C l}}{p_{H_{2}}}$ mit $\alpha$, so wird

$$
\frac{1}{K_{1}}=\frac{p_{H C l}^{2}}{p_{H_{2}}}=\frac{\alpha^{2}}{1+\alpha} .
$$

In Fig. 3 ist $\log \frac{p_{\mathrm{HCl}}^{2}}{p_{\mathrm{H}_{2}}}$ in Abhängigkeit von $\frac{1}{T} \cdot 10^{4}$ aufgetragen.

1) Nach W. Nernst, Z. Elektrochem. 15, 687. 1909. 
Für das $\mathrm{FeCl}_{3}$ gelten diese Beziehungen nicht, da dieses Salz bei der Versuchstemperatur $T=564^{\circ}$ einen nicht mehr zu vernachlässigenden Dampfdruck aufwies. In diesem Falle wird so verfahren, dass man von $1 \mathrm{Atm}$. die Dampfspannung des $\mathrm{FeCl}_{3}$ für die Temperatur $T=564^{\circ}$ in Abrechnung bringt und den Rest im Verhältnis $\frac{p_{H C l}}{p_{H_{2}}}$ teilt. Die neuerdings von JELLINEK und RUdAT nach einer modifizierten Mitführungsmethode gemessenen Dampfspannungen des $\mathrm{FeCl}_{3}$ sollen hier veröffentlicht werden ${ }^{1}$ ).

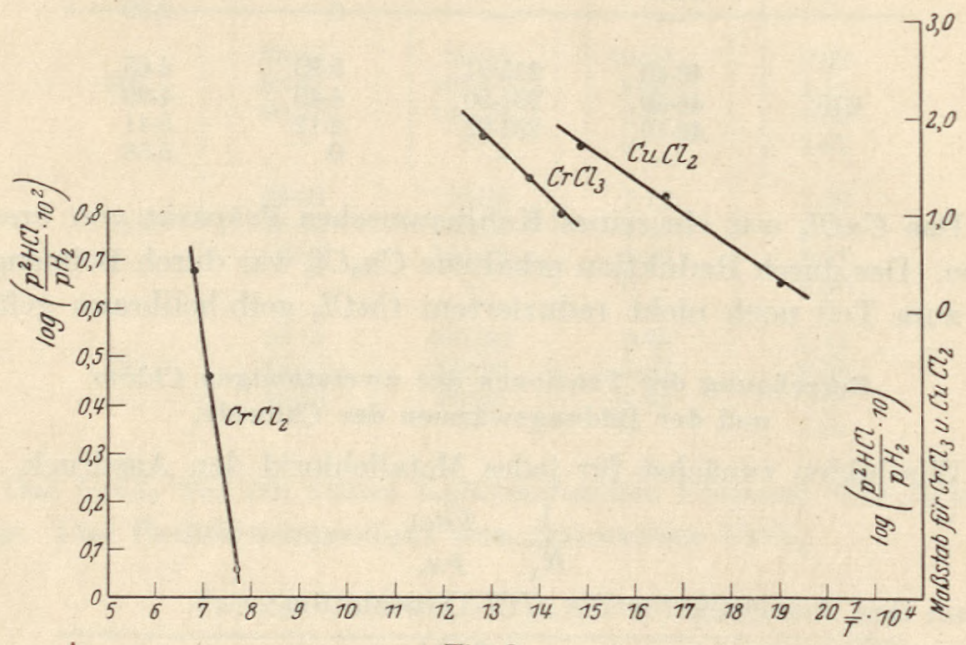

Fig. 3.

In der Tabelle 9 sind eingetragen: die Versuchstemperatur $T$, ferner die durch Extrapolation auf die Strömungsgeschwindigkeit Null ermittelten Dampfspannungen in Atmosphären ( $p$ in Atm.) und die dekadischen Logarithmen dieser Dampfspannungen $\log p_{\text {Atm. }}$.

Tabelle 9. Dampfdrucke von $\mathrm{FeCl}_{3}$.

\begin{tabular}{c|r|r|r}
\hline$T$ & $\begin{array}{c}p \\
\mathrm{~mm} H\end{array}$ & $\begin{array}{c}p \\
\text { in Atm. }\end{array}$ & $\log p_{\text {Atm. }}$. \\
\hline 473 & 9.1 & 0.012 & -1.9208 \\
513 & 67.0 & 0.088 & -1.0555 \\
533 & 181.0 & 0.237 & -0.6253 \\
553 & 387.0 & 0.508 & -0.2941
\end{tabular}

1) Zur Methode vgl. Jellinek und Rosner, Z. physikal. Chem. (A) 143, 51. 1929. Jellinek und Rudat, Z. physikal. Chem. (A) 143, 55. 1929. 
Das verwendete $\mathrm{FeCl}_{3}$ war ein reines Kahlbaumsches Präparat. Es wurde zur nochmaligen Reinigung im sauerstofffreien Stickstoffstrom sublimiert.

In Fig. 4 sind die $\log p_{\text {Atm. }}$ gegen $\frac{1}{T} \cdot 10^{4}$ aufgetragen. Es ergibt sich eine gerade Linie, was eine konstante äussere Verdampfungswärme fordert. Diese ergibt sich nach der Gleichung $\log p_{1}-\log p_{2}=-\frac{\lambda}{4 \cdot 57}\left(\frac{1}{T_{1}}-\frac{1}{T_{2}}\right)$ zu $24780 \mathrm{cal}$ pro $\mathrm{Mol} \mathrm{FeCl}_{3}$.

Wir teilen bei dieser Gelegenheit auch die von JELLINEK

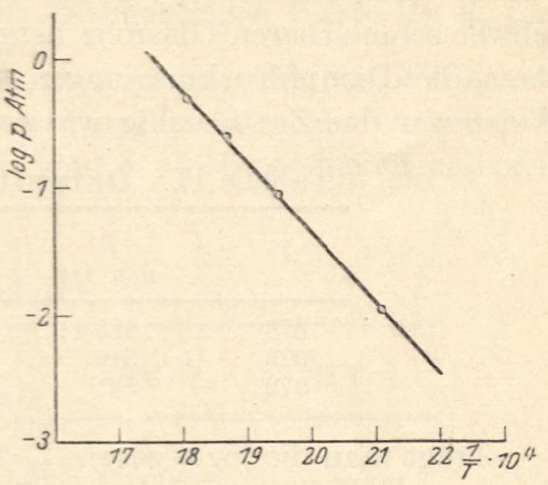

Fig. 1. und Rudat gemessenen Dampfdrucke des $\mathrm{CrCl}_{3}$ und $\mathrm{ZnCl}_{2}$ mit. Das verwendete $\mathrm{CrCl}_{3}$ war ein reines Kahlbaumsches Präparat.

\begin{tabular}{c|c|c|c} 
Tabelle 10. Dampfdrucke & von $\mathrm{CrCl}_{3}$. \\
\hline$T$ & $\begin{array}{c}p \\
\text { mm } \mathrm{Hg}\end{array}$ & $\begin{array}{c}\text { in Atm. } \\
\text { in }\end{array}$ & $\log p_{\text {Atm. }}$. \\
\hline 973 & 14.2 & 0.0187 & -1.726 \\
1073 & 47.0 & 0.0618 & -1.209 \\
1173 & 140.2 & 0.1845 & -0.734 \\
1273 & 278.2 & 0.366 & -0.436
\end{tabular}

In Fig. 5 sind wieder $\log$ $p_{\text {Atm. }}$ gegen $\frac{1}{T} \cdot 10^{4}$ eingetragen. Es ergibt sich wieder eine gerade Linie. Die molare Verdampfungswärme beträgt $24600 \mathrm{cal}$, also fast ebensoviel als pro $1 \mathrm{Mol}$ $\mathrm{FeCl}_{3}$. Wie aus den in Tabelle 6 gegebenen Temperaturdaten ersichtlich ist, braucht ein Dampfdruck des $\mathrm{CrCl}_{3}$ dort nicht berücksichtigt $\mathrm{zu}$ werden. Wie

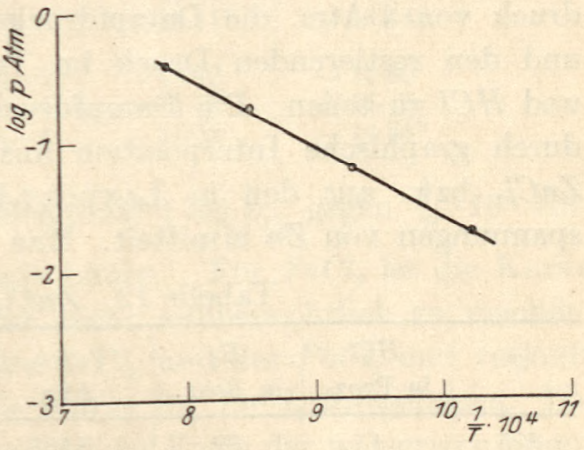

Fig. 5. weiter aus Fig. 6 ersichtlich, ist bei den Daten von Tabelle 10 eine $\mathrm{Cl}_{2}$-Abspaltung des $\mathrm{CrCl}_{3}$ noch nicht merklich. 
Das zu Dampfdruckmessungen verwendete Zinkchlorid wurde synthetisch aus Zinkdampf, der mittels Kahlbaumschen analysenreinem Zink entwickelt wurde, und Chlorgas direkt in demselben schwer schmelzbaren Glasrohr hergestellt, in welchem nach der Synthese die Dampfdruckmessungen vorgenommen wurden. Auf diese Weise war das $\mathrm{ZnCl}_{2}$ völlig wasserfrei.

\begin{tabular}{c|c|c|c}
\multicolumn{4}{c}{ Tabelle 11. Dampfdrucke von $\mathrm{ZnCl}_{2}}$. \\
\hline$T$ & $\begin{array}{c}p \\
\text { mm Hg}\end{array}$ & $\begin{array}{c}p \\
\text { in Atm. }\end{array}$ & $\log p_{\text {Atm. }}$. \\
\hline 873 & 125.4 & 0.165 & -0.7825 \\
923 & 316 & 0.416 & -0.3809 \\
973 & 591 & 0.778 & -0.1090
\end{tabular}

Trägt man die $\log p$ gegen $\frac{1}{T} \cdot 10^{4}$ auf, so erhält man eine gerade Linie. Die Verdampfungswärme von $1 \mathrm{Mol} \mathrm{ZnCl}_{2}$ ergibt sich nach

$$
\log p_{1}-\log p_{2}=-0 \cdot 1090+0.7825=-\frac{\lambda}{4 \cdot 571} \cdot(0 \cdot 001028-0.001146)
$$

zu $26080 \mathrm{cal}$.

Die nunmehr bekannten Dampfspannungen von $\mathrm{ZnCl}_{2}$ ermöglichen es nun auch, das von A. B. BAGdASARIAN ${ }^{1}$ ) gemessene $H_{2}$-Reduktionsgleichgewicht des $\mathrm{ZnCl}_{2}$ heranzuziehen. Man muss dabei beachten, dass dieser Autor bei $1 \mathrm{Atm}$. Gesamtdruck nur die volumprozentische Zusammensetzung des aus $\mathrm{H}_{2}$ und $\mathrm{HCl}$ bestehenden abziehenden Gasgemisches bestimmt hat. Um die Gleichgewichtspartialdrucke von $\mathrm{H}_{2}$ und $\mathrm{HCl}$ zu erhalten, hat man von dem Gesamtdruck von $1 \mathrm{Atm}$. die Dampfdrucke von $\mathrm{ZnCl}_{2}$ und $\mathrm{Zn}$ abzuziehen und den restierenden Druck im Verhältnis der Volumprozente $\mathrm{H}_{2}$ und $\mathrm{HCl}$ zu teilen. Die Dampfdrucke von $\mathrm{ZnCl}_{2}$ und $\mathrm{Zn}$ haben wir durch graphische Interpolation aus obigen Dampfspannungen von $\mathrm{ZnCl}_{2}$ bzw. aus den in Landolt-Bönnstern ${ }^{2}$ ) gegebenen Dampfspannungen von $Z n$ ermittelt. Man erhält so Tabelle 12.

Tabelle 12. $\mathrm{ZnCl}_{2}+\mathrm{H}_{2} \rightleftarrows \mathrm{Zn}+2 \mathrm{HCl}$.

\begin{tabular}{c|c|c|c|c|c|c}
\hline$T$ & $\begin{array}{c}\mathrm{HCl} \\
\text { in Proz. }\end{array}$ & $\begin{array}{c}\mathrm{H}_{2} \\
\text { in Proz. }\end{array}$ & $\begin{array}{c}p_{\mathrm{ZnCl}_{2}} \\
\text { Atm. }\end{array}$ & $\begin{array}{c}p_{\mathrm{Zn}_{2}} \\
\text { Atm. }\end{array}$ & $\begin{array}{c}p_{\mathrm{HCl}} \\
\text { Atm. }\end{array}$ & $p_{\mathrm{H}_{2}}$ \\
\hline 875 & 1 & 99 & $0.16 \tilde{5}$ & 0.015 & 0.0082 & 0.812 \\
923 & 3 & 97 & 0.416 & 0.033 & $0.016 \tilde{2}$ & 0.5334 \\
980 & 5 & 95 & 0.871 & 0.074 & 0.0028 & 0.052
\end{tabular}

1) A. B. Bagdasarian, Trans. Amer. Elektrochem. Soc. 51, 449. 1927.

2) LAindolt-Börnstein, Physikalisch-chemische Tabellen. 5. Aufl. 
In Tabelle 13 sind nun die Chlortensionen der besprochenen Metallchloride verzeichnet, und zwar sind in Tabelle 13 für jedes Metallchlorid die Werte $\log \frac{1}{K_{1}}$, die dazugehörigen Werte $\log K_{2}$ und $\log p_{\mathrm{Cl}_{2}}$ berechnet. Die entsprechenden Werte für das $\mathrm{FeCl}_{2}$ und $\mathrm{ZnCl}_{2}$ sind, wie erwähnt, aus den Versuchsdaten der Arbeit von A. B. BAGDASARIAN ${ }^{1}$ ) errechnet, sie sind der Vollständigkeit halber in die Tabelle aufgenommen worden.

Tabelle 13.

\begin{tabular}{|c|c|c|c|c|}
\hline Stoff & $T$ & $\log \frac{1}{K_{1}}$ & $\log K_{2}$ & $\log p_{C l_{2}}$ \\
\hline $\begin{array}{c}\mathrm{FeCl}_{3} \\
\mathrm{zu} \\
\mathrm{FeCl}\end{array}$ & $\tilde{5} 64$ & $0.2642-3$ & -17.825 & -20.561 \\
\hline $\begin{array}{c}\mathrm{FeCl} \\
\mathrm{zu}_{2} \\
\mathrm{Fe}\end{array}$ & $\begin{array}{r}975 \\
1073 \\
1198 \\
1205\end{array}$ & $\begin{array}{l}0.8062-2 \\
0 \cdot 4728-1 \\
0 \cdot 8848-1 \\
0.9101-1\end{array}$ & $\begin{array}{r}-10 \cdot 567 \\
-\quad 9 \cdot 648 \\
-\quad 8 \cdot 693 \\
-\quad 8 \cdot 646\end{array}$ & $\begin{array}{r}-11.761 \\
-10.175 \\
-\quad 8.808 \\
-8.736\end{array}$ \\
\hline $\begin{array}{l}\mathrm{CrCl}_{3} \\
\mathrm{zu} \\
\mathrm{CrCl}_{2}\end{array}$ & $\begin{array}{l}689 \\
718 \\
783\end{array}$ & $\begin{array}{l}0.0204 \\
0.4323 \\
0.8082\end{array}$ & $\begin{array}{l}-14.72 \\
-14.14 \\
-13.019\end{array}$ & $\begin{array}{r}-14 \cdot 70 \\
-13 \cdot 71 \\
-12 \cdot 21\end{array}$ \\
\hline $\begin{array}{c}\mathrm{CrCl}_{2} \\
\mathrm{zu}^{2} \\
\mathrm{Cr}\end{array}$ & $\begin{array}{l}1294 \\
1408 \\
1465\end{array}$ & $\begin{array}{l}0.0610-2 \\
0.4606-2 \\
0.6833-2\end{array}$ & $\begin{array}{r}8.081 \\
-\quad 7.466 \\
-\quad 7.190\end{array}$ & $\begin{array}{r}10.017 \\
-\quad 9.005 \\
-8.507\end{array}$ \\
\hline $\begin{array}{c}\mathrm{CuCl}_{2} \\
\mathrm{zu} \\
\mathrm{Cu}_{2} \mathrm{Cl}_{2}\end{array}$ & $\begin{array}{l}524 \\
598 \\
670\end{array}$ & $\begin{array}{l}0 \cdot 3221-1 \\
0 \cdot 2049 \\
0 \cdot 6747\end{array}$ & $\begin{array}{l}-19 \cdot 157 \\
-16 \cdot 864 \\
-10 \cdot 117\end{array}$ & $\begin{array}{l}-19 \cdot 83 \tilde{5} \\
-16 \cdot 6 \tilde{9} \\
-14 \cdot 442\end{array}$ \\
\hline $\begin{array}{c}\mathrm{ZnCl}_{2} \\
\mathrm{zu} \\
\mathrm{Zn}\end{array}$ & $\begin{array}{l}875 \\
923 \\
980\end{array}$ & $\begin{array}{l}0.9180-5 \\
0.707 \tilde{5}-4 \\
0.1784-4\end{array}$ & $\begin{array}{r}-11.72 \\
-11.13 \\
-10.52\end{array}$ & $\begin{array}{l}-15 \cdot 80 \\
-14 \cdot 42 \\
-14 \cdot 34 ?\end{array}$ \\
\hline
\end{tabular}

In Fig. 6 sind für alle Metallchloride $\left.{ }^{2}\right) \log p_{\mathrm{Cl}_{2}}$ gegen $\frac{1}{T} \cdot 10^{4}$ aufgetragen. Die Kurven sind gerade Linien. Für $\mathrm{FeCl}_{2}$ ist die Kurve nicht eingezeichnet, um die Figur nicht unübersichtlich zu machen. Sie liegt zwischen den Kurven des $\mathrm{CrCl}_{2}$ und des $\mathrm{PbCl}_{2}$ und verläuft parallel zu diesen. Ebenso ist die Kurve für $\mathrm{ZnCl}_{2}$ fortgelassen. Die Metalle ordnen sich im allgemeinen in der Folge der wässerigen Span-

1) A. B. Bagdasarian, Trans. Amer. Elektrochem. Soc. 51, 449. 1927.

2) Vgl. die Arbeiten von JeLLINEK und ULOTH, ferner von JeLLINEK und Rudat, loc. cit. 
nungsreihe. Die auffallende Stellung von $N i$ und $C o$ ist schon in der Arbeit von JELLINEK und ULOTH festgestellt worden. $\mathrm{CuCl}_{2}$ und $\mathrm{CrCl}_{3}$ haben eine stärkere Chlortension als $\mathrm{Cu}_{2} \mathrm{Cl}_{2}$ und $\mathrm{CrCl}_{2}$.

Der Wert für die Chlortension des $\mathrm{ZnCl}_{2}$ bei der höchsten von BAGDASARIAN gemessenen Temperatur von $T=980$, bei welcher $p_{\mathrm{ZnCl} l_{2}}$ bereits $0.871 \mathrm{Atm}$. und $p_{Z n}=0.074 \mathrm{Atm}$. ist, wird offenbar sehr fehlerhaft sein und soll daher aus den weiteren Betrachtungen fortbleiben.

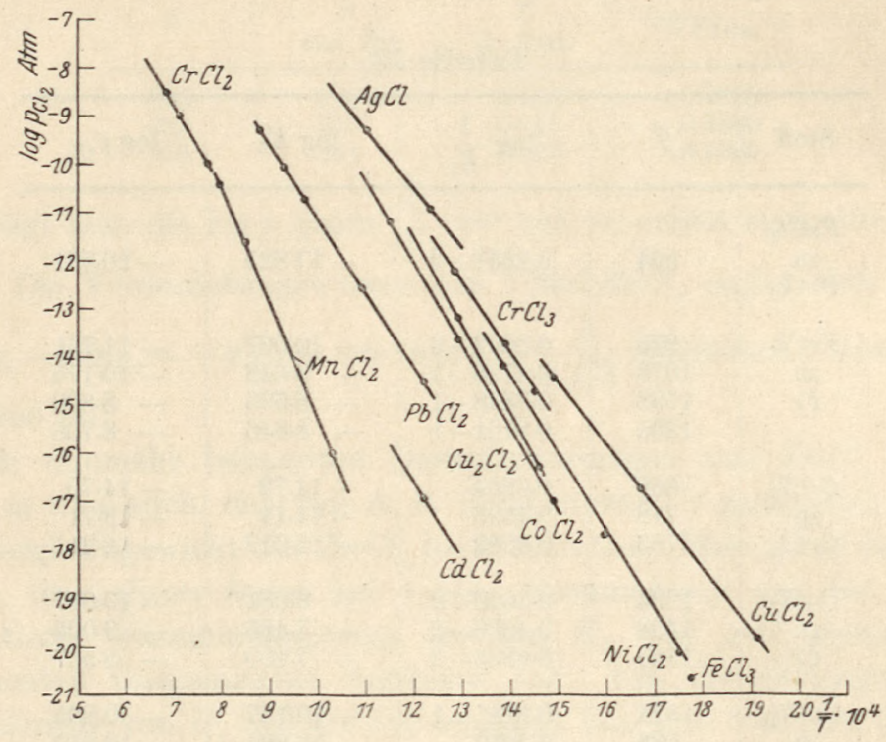

Fig. 6.

Die Chlortensionen des $\mathrm{ZnCl}_{2}$ lassen sich auch aus den Messungen der EMK für Ketten mit geschmolzenem $\mathrm{ZnCl}_{2}$ von R. LoRENz und R. SuCHY ${ }^{1}$ ) durch graphische Interpolation berechnen. Es ergibt sich dann folgende Tabelle 14.

Tabelle 14.

\begin{tabular}{c|c|c}
\hline$T$ & $\begin{array}{c}\log p_{\mathrm{Cl}_{2} \text { Atm. }} \\
\text { B.GDASARIAN }\end{array}$ & $\begin{array}{c}\log p_{\mathrm{Cl}_{2}} \\
\text { LORENZ und SUCHY }\end{array}$ \\
\hline 875 & $-15 \cdot 80$ & -17.5 \\
923 & $-14 \cdot 42$ & $-16 \cdot 2$ \\
980 & $-14 \cdot 34$ & -14.9
\end{tabular}

1) R. LoRenz und R. Suchy, Z. f. anorg. Chem. 27, 152. 1901. 
Die nach beiden Methoden gemessenen Chlortensionen zeigen also einen beträchtlichen Unterschied, auch wenn man von dem Wert bei $T=980$ absieht.

Die Bildungswärme der Chloride ergibt sich aus der Gleichung

$$
\log p_{C_{2}}^{\prime}-\log p_{C_{2}}^{\prime \prime}=-\frac{Q}{4 \cdot 57}\left(\frac{1}{T^{\prime \prime}}-\frac{1}{T^{\prime \prime}}\right) \text {. }
$$

In Tabelle 15 sind die molaren Bildungswärmen $Q$ in Calorien bezogen auf die mittlere Temperatur $T_{m}$ berechnet. Zum Vergleich sind in der letzten Spalte der Tabelle calorimetrisch gemessene Bildungswärmen eingetragen.

Tabelle 15 .

\begin{tabular}{|c|c|c|c|c|c|}
\hline Stoff & $T$ & $\log p_{C_{2}}$ & $T_{m}$ & $Q$ in cal & $\begin{array}{l}Q \text { in cal } \\
\text { calorimetrisch }^{1} \text { f für } \\
\text { Zimmertemperatur }\end{array}$ \\
\hline $\begin{array}{c}\mathrm{Fe}+1 \cdot \tilde{\mathrm{CCl}} \mathrm{l}_{2} \\
=\mathrm{FeCl} l_{3}\end{array}$ & - & - & - & - & 96040 \\
\hline $\begin{array}{l}\mathrm{Fe}+\mathrm{Cl}_{2} \\
=\mathrm{FeCl}\end{array}$ & $\begin{array}{r}975 \\
1205\end{array}$ & $\begin{array}{r}-11.761 \\
-\quad 8.736\end{array}$ & 1090 & $69130^{2}$ ) & 82050 \\
\hline $\begin{array}{c}2 \mathrm{CrCl}_{2}+\mathrm{Cl}_{2} \\
=2 \mathrm{CrCl}_{3}\end{array}$ & $\begin{array}{l}689 \\
783\end{array}$ & $\begin{array}{l}-14 \cdot 70 \\
-12 \cdot 21\end{array}$ & 736 & 66950 & $\begin{array}{c}56700 \\
\left(\mathrm{CrCl}_{2} \mathrm{aq}+1 / 2 \mathrm{Cl}_{2}\right. \\
\left.=\mathrm{CrCl} l_{3} \cdot \mathrm{aq}\right)\end{array}$ \\
\hline $\begin{array}{l}\mathrm{Cr}+\mathrm{Cl}_{2} \\
=\mathrm{CrCl}_{2}\end{array}$ & $\begin{array}{l}1294 \\
1465\end{array}$ & $\begin{array}{r}-10.017 \\
-\quad 8.507\end{array}$ & 1379 & 76680 & - \\
\hline $\begin{array}{c}\mathrm{Cr}+1.5 \mathrm{Cl}_{2} \\
=\mathrm{CrCl}_{3}\end{array}$ & - & - & 1058 & $\begin{array}{r}76680 \\
+33500 \\
110180\end{array}$ & - \\
\hline $\begin{array}{c}C u_{2} \mathrm{Cl}_{2}+\mathrm{Cl}_{2} \\
=2 \mathrm{CuCl}_{2}\end{array}$ & $\begin{array}{l}524 \\
670\end{array}$ & $\begin{array}{l}-19 \cdot 835 \\
-14.442\end{array}$ & 597 & 58700 & - \\
\hline $\begin{array}{c}\mathrm{Zn}+\mathrm{Cl}_{2} \\
=\mathrm{ZnCl}_{2}\end{array}$ & $\begin{array}{l}875 \\
923\end{array}$ & $\begin{array}{l}-15 \cdot 80 \\
-14.42\end{array}$ & 900 & $\left.105200^{2}\right)$ & 98700 \\
\hline
\end{tabular}

Die Wärmetönung bei der Verwandlung von $\mathrm{CrCl}_{2}$ in $\mathrm{CrCl}_{3}$ beträgt etwa die Hälfte der Wärmetönung bei der Verwandlung von $\mathrm{Cr}$ in $\mathrm{CrCl}_{2}$, so dass für die Bildungswärme des $\mathrm{CrCl}_{3} 110200 \mathrm{cal}$ gefunden werden. Aus den bei Zimmertemperatur beobachteten Wärmetönungen:

1) Die Daten sind den Tabellen von Landolt-Börnstern entnommen. 2) Aus den Daten der Arbeit von Bagdasarian (loc. cit.) berechnet. 


$\begin{array}{lc}2 \mathrm{Cu}+2 \mathrm{Cl}_{2}=2 \mathrm{CuCl}_{2}+103000 \mathrm{cal} \text { (Thомsen) } \\ \text { und } & 2 \mathrm{Cu}+\mathrm{Cl}_{2}=\mathrm{Cu}_{2} \mathrm{Cl}_{2}+65740 \mathrm{cal} \text { (THomseN) } \\ \text { folgt für } & \mathrm{Cu}_{2} \mathrm{Cl}_{2}+\mathrm{Cl}_{2}=2 \mathrm{CuCl}_{2}\end{array}$

der Wert von nur 37260 cal, während aus obigen Daten der Wert von $58700 \mathrm{cal}$ folgte. Vielleicht ist die Bildungswärme von $\mathrm{CuCl}_{2}$ zu niedrig beobachtet.

\section{Die heterogenen Gleichgewichte der Metallfluoride mit Chlorwasserstoff.}

Die bisher noch nicht näher untersuchten schwierig zu messenden Gleichgewichte vom Typus

$$
\mathrm{MeF}_{2}+2 \mathrm{HCl} \rightleftarrows \mathrm{MeCl}_{2}+2 \mathrm{HF}
$$

sollten hier erstmalig quantitativ gemessen werden ${ }^{1}$ ). Zur Messung wird die Strömungsmethode verwendet. Wegen der Anwesenheit von $\mathrm{HCl}$ - und $\mathrm{HF}$-Gas im Reaktionsrohr konnten für die heisse Zone nur Apparateteile aus Silber, für die kalte Zone nur solche aus Kupfer und paraffiniertem Glas gewählt werden.

\section{Apparatur und Arbeitsweise.}

Der Chlorwasserstoff wird in einer von F.W. Küster und R. ABEgG angegebenen Apparatur entwickelt ${ }^{2}$ ). Das Gas passiert einen mit konzentrierter $\mathrm{H}_{2} \mathrm{SO}_{4}$ halb gefüllten Zylinder, der als Druckregulator wirkt ${ }^{3}$ ), durchströmt dann ein Kapometer mit konzentrierter $\mathrm{H}_{2} \mathrm{SO}_{4}$ als Sperrflüssigkeit und wird vor Eintritt in das Reaktionsrohr in einem $\mathrm{CaCl}_{2}$-Turm getrocknet.

Das Reaktionsrohr besteht, wie schon oben erwähnt, aus Feinsilber ( $850 \mathrm{~mm}$ lang, $1 \mathrm{~mm}$ Wandstärke). Auf eine Länge von $500 \mathrm{~mm}$ hat es eine lichte Weite von $14 \mathrm{~mm}$, das Rohr verjüngt sich dann und hat auf seine restliche Länge von $350 \mathrm{~mm}$ eine lichte Weite von $4 \mathrm{~mm}$. Diese Form wurde gewählt, um die Zeit des Ausspülens vor jedem Versuch zu verkürzen und damit die Substanz zu schonen (vgl. weiter unten).

In dem Reaktionsrohr befindet sich ein Feinsilberschiffchen für 10 bis $15 \mathrm{~g}$ Substanz.

1) Betreffs qualitativer Versuche vgl. KüHLmans, Ann. Physik 10, 618. 1827. H. Sainte-Clatre Deville, Liebigs Ann. 101, 197. 1856. C. r. 43, 970. 1856. J. B. 1856, 301. C. Poulenc, C. r. 114, 1426. 1892. 115, 942. 1892. Chem. Ztrblt. 1892, II, 205. Moissan, Chimie Minérale 4, 163, 328, 329. ${ }^{2}$ ) F. W. Küster und R. ABEGG, Z. chem. Apparatekunde 1, 89. $1906 . \quad$ 3) Vgl. weiter unten. 
Der $\mathrm{HCl}$ gelangt auf seinem weiteren Wege aus dem Trockenturm durch ein Glasrohr mit eingeschliffenem Hahn $h_{1}$ in den weiteren Teil des Silberrohres, den eigentlichen Reaktionsraum und setzt sich hier in der heissen Zone mit dem Metallfluorid um. Die Verbindung des Glasrohres mit dem Silberrohr geschieht vermittels eines Kautschukstopfens.

Als Ofen dient ein mit Chromnickeldraht bewickeltes $600 \mathrm{~mm}$ langes, $30 \mathrm{~mm}$ weites Porzellanrohr. In dieses ist das Reaktionsrohr derart eingebaut, dass es zu beiden Seiten $120 \mathrm{~mm}$ aus dem Ofen herausragt. Neben dem Reaktionsrohr liegt ein Thermoelementschutzrohr (450 mm lang, $7 \mathrm{~mm}$ weit und einseitig geschlossen) aus Feinsilber und führt bis in die Ofenmitte hinein.

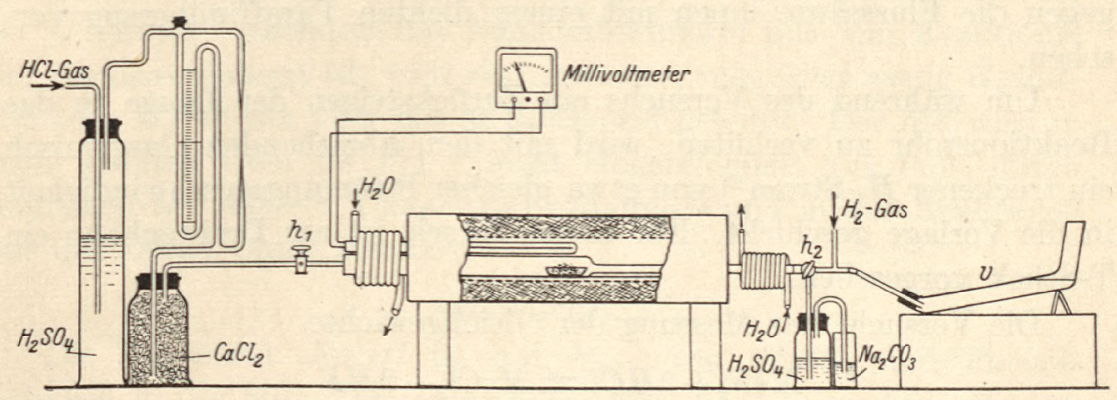

Fig. 7.

Die aus dem Heizrohr beiderseits herausragenden Silberrohrenden werden mit Bleirohr umwickelt, durch welches zur Kühlung Wasser hindurchgeschickt wird.

Zur Temperaturmessung wird ein geeichtes $P t / P t$ - $R h$-Thermoelement von Heraeus benutzt ${ }^{1}$ ); die EMK zeigt ein Millivoltmeter von Siemens \& Halske an.

Wie nun vergleichende Versuche mit zwei Thermoelementen ergaben, hielt sich die Temperatur in beiden Silberrohren über eine mittlere Strecke von etwa $15 \mathrm{~cm}$ konstant. Bei den endgültigen Versuchsreihen befand sich das Substanzschiffchen innerhalb dieser Zone, das Thermoelement lag in deren Mitte. Es zeigte sich ferner, dass die Thermoelementtemperatur im Schutzrohr um $7^{\circ}$ höher lag, als im Reaktionsrohr. Dieser Unterschied wurde bei den Temperaturangaben berücksichtigt. Die Genauigkeit der Messung betrug für alle Temperaturangaben $1 \%$.

1) Vgl. oben. 
Das entstehende Gasgemisch $(\mathrm{HF}+\mathrm{HCl})$ verlässt das Reaktionsrohr am eng ausgezogenen Ende und kann vermöge eines dicht angesetzten kupfernen Dreiweghahns $h_{2}{ }^{1}$ ) entweder in eine mit ${ }^{1 / 10}$ norm. $\mathrm{NaOH}$ gefüllte Absorptionsvorlage $v$ oder zwecks Vernichtung in eine Flasche mit konzentrierter $\mathrm{H}_{2} \mathrm{SO}_{4}$ und dann in Sodalösung geleitet werden.

Das Absorptionsgefäss ist ein horizontal liegendes Glasrohr (350 mm lang, $20 \mathrm{~mm}$ weit) mit einseitig ansteigendem Schenkel. Am Einleitungsende ist ein Platinrohr (mit Marineleim) eingekittet, das am Dreiweghahn wieder vermittels einer dort angelöteten zweiten Muffe angeschlossen werden kann. Das Glasgefäss wird zum Schutz gegen die Flusssäure innen mit einem dichten Paraffinüberzug versehen.

Um während des Versuchs ein Zurücksteigen der Lauge in das Reaktionsrohr zu verhüten, wird mit dem abziehenden Gasgemisch ein trockener $\mathrm{H}_{2}$-Strom ${ }^{2}$ ) von etwa gleicher Strömungsgeschwindigkeit in die Vorlage geschickt. Für diesen Zweck ist am Dreiweghahn ein T-Stück vorgesehen.

Die Versuche zur Messung der Gleichgewichte

$$
\mathrm{MeF}_{2}+2 \mathrm{HCl} \rightleftarrows \mathrm{MeCl}_{2}+2 \mathrm{HF}
$$

werden nun folgendermassen ausgeführt. Nachdem die Temperatur des Ofens konstant geworden ist, lässt man den $\mathrm{HCl}$ durch entsprechendes Öffnen der Hähne $h_{1}$ und $h_{2}$ über das Metallfluorid in die $\mathrm{H}_{2} \mathrm{SO}_{4}$-Flasche strömen und reguliert die Gasgeschwindigkeit. Durch Verschieben der Einströmungskapillare des Glaszylinders lässt sich eine konstant bleibende Strömungsgeschwindigkeit einstellen, die ảm empirisch geeichten Kapometer abgelesen wird. Nun öffnet man den $H_{2}$-Kipp und lässt das Gas durch das T-Stück langsam nach aussen strömen. Bei strömendem Wasserstoff wird die gefüllte Absorptionsvorlage am freien Ende des T-Stückes mit Marineleim angeschlossen.

Inzwischen ist das Reaktionsrohr genügend ausgespült, d. h. die gesamte Luft aus dem System verdrängt und das zu messende Gasgemisch mit konstanter Geschwindigkeit durch das Reaktionsrohr geströmt. Man öffnet jetzt den Dreiweghahn zur Vorlage und lässt

1) An den Dreiweghahn ist eine kupferne Muffe angelötet, die über das Silberrohrende geschoben wird. Zur Abdichtung wird Marineleim verwendet. ${ }^{2}$ ) Der Wasserstoff wird in einem Kipp entwickelt, mit konzentriertem $\mathrm{H}_{2} \mathrm{SO}_{4}$ und $\mathrm{CaCl}_{2}$ getrocknet. 
für eine bestimmte Zeit das Gas von der Lauge absorbieren ${ }^{1}$ ). Die Versuchsdauer wird auf $1 \%$ genau ermittelt. Aus der Gesamtgasmenge $(H F+H C l)$ und der Versuchsdauer ergibt sich durch Division die auf etwa $1 \%$ genaue Strömungsgeschwindigkeit.

Es ist bei den endgültigen Versuchen unbedingt jede Feuchtigkeit im Reaktionsrohr zu vermeiden, da sonst undefinierbare Mengen $\mathrm{HCl}$ und $H F$-Gas der Messung entzogen werden.

Da die feuchten Metallfluoride leicht Oxysalze bilden, werden sie vor Beginn der Versuche getrocknet. Die Trocknung geschieht derart, dass man das Metallfluorid mit trockenem Ammoniumfluorid innig vermischt und im Reaktionsrohr auf höhere Temperatur $\left(400^{\circ}\right.$ bis $500^{\circ} \mathrm{C}$ ) erhitzt. Nachdem das Ammoniumfluorid aus dem Reaktionsrohr heraussublimiert ist, wird ein trockener, möglichst wenig $\mathrm{H}_{2}$ enthaltender $H F$-Strom über das Metallfluorid geleitet. Der $H F$ wird in einem zweiten Ofen (in Fig. 7 nicht eingezeichnet) aus $P b F_{2}$ durch Reduktion bei höherer Temperatur erhalten ${ }^{2}$ ) und in das Reaktionsrohr des ersten Ofens geschickt.

\section{Analytisches.}

Die $1 / 10$ norm. $\mathrm{NaOH}$ ist mit $1 / 10$ norm. $\mathrm{H}_{2} \mathrm{SO}_{4}$ und Phenolphthalein in der Siedehitze eingestellt. Für einen Versuch werden 50 bis $60 \mathrm{~cm}^{3} 1 / 10$ norm. $\mathrm{NaOH}$ in die Vorlage hineinpipettiert. Nach Beendigung des Versuchs füllt man den Inhalt der Vorlage in einem Messkolben auf $150 \mathrm{~cm}^{3}$ auf und titriert davon $50 \mathrm{~cm}^{3}$ in der Siedehitze mit $1 / 10$ norm. $\mathrm{H}_{2} \mathrm{SO}_{4}$ und Phenolphthalein (Platinschale). Man erhält damit die Kubikzentimeter Gas $(H F+H C l)$. Zu der nun neutralisierten Lösung gibt man nach dem Erkalten einige Tropfen einer gesättigten $\mathrm{K}_{2} \mathrm{CrO}_{4}$-Lösung und titriert nach MоHR mit ${ }^{1 / 10}$ norm. $\mathrm{AgNO}_{3}$-Lösung. Man findet somit die Kubikzentimeter $\mathrm{HCl}$. Aus der Differenz beider Analysenresultate ergeben sich die Kubikzentimeter $H F$. Die Analysen waren auf $0 \cdot 1$ bis $0 \cdot 2 \%$ genau.

In dem Gleichgewicht

\section{Versuchsergebnisse.}

$$
\mathrm{MeF}_{2}+2 \mathrm{HCl} \rightleftarrows \mathrm{MeCl}_{2}+2 \mathrm{HF}
$$

sind bei drei Bestandteilen und drei Phasen die Anzahl der Frei-

1) Es wurden jeweils etwa 100 bis $120 \mathrm{~cm}^{3}$ Gas aufgefangen, so dass man bei empirisch bekannter Strömungsgeschwindigkeit die Versuchsdauer von vornherein festlegen konnte. $\quad{ }^{2}$ ) JELLINEK und RUDAT, Z. anorgan. u. allgem. Chem. 175, 281. 1928. 
heiten 2. Wenn wir über die Temperatur und den Druck $p$ (1 Atm.) verfügen, dann ist für das Gleichgewicht alles festgelegt. Nach dem Massenwirkungsgesetz gilt

$$
\frac{p_{\mathrm{MeCl}_{2}} \cdot p_{H F}^{2}}{p_{\mathrm{HeF}_{2}} \cdot p_{H C l}^{2}}=K \text { bzw. } \frac{p_{H F}^{3}}{p_{H C l}^{2}}=K^{\prime} \text { oder } \frac{p_{H F}}{p_{H C l}}=K^{\prime \prime},
$$

da wir den Dampfdruck der Bodenkörper als konstant betrachten können. In keinem der folgenden Gleichgewichte war eine Abhängigkeit der Zusammensetzung der Gasphase vom Mischungsverhältnis der Bodenkörper zu beobachten ${ }^{1}$ ).

In den folgenden Tabellen sind für jedes Metallfluorid eingetragen: die absolute Versuchstemperatur $T$, die durch Titration gefundenen

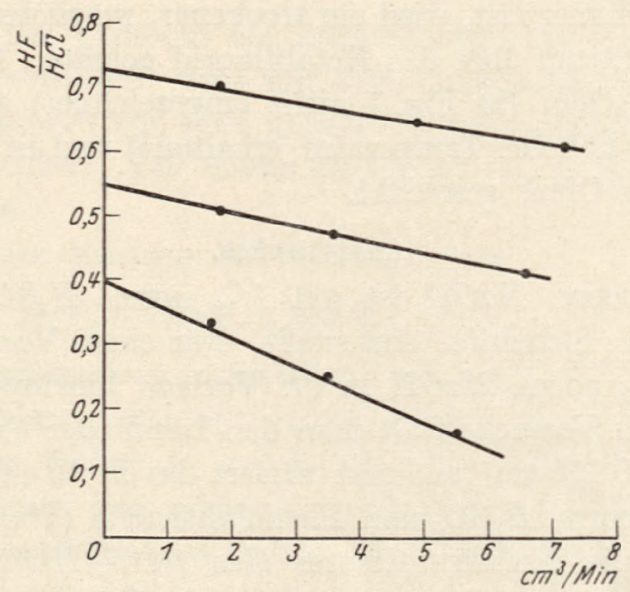

Fig. 8.

Kubikzentimeter $\mathrm{HCl}$ und die Kubikzentimeter $\mathrm{HF}$, die Strömungsgeschwindigkeit, d. h. der Quotient aus den Kubikzentimetern Gas $(\mathrm{HF}+\mathrm{HCl})$ und der Versuchsdauer in Minuten $\left(\mathrm{cm}^{3} / \mathrm{Min}.\right)$ und der Quotient aus den Kubikzentimetern $\mathrm{HF}$ und den Kubikzentimetern $\mathrm{HCl}$ $\left(\frac{H F}{H C l}\right)$. Durch Extrapolation wird der Wert $\frac{H F}{H C l}$ für die Strömungsgeschwindigkeit Null ermittelt. Als Beispiel für die Sicherheit der Extrapolation sind die Strömungskurven für $\mathrm{CoF}_{2}$ in Fig. 8 gegeben.

1) In der Arbeit von Jellinek und Itelsohn (Z. anorgan. u. allgem. Chem. 155, 130. 1926) war dagegen bei der Reaktion $\mathrm{KBr}+\mathrm{HCl} \rightleftarrows \mathrm{KCl}+\mathrm{HBr}$ Mischkristallbildung beobachtet worden. 
Tabelle 16. $\mathrm{CoF}_{2}+2 \mathrm{HCl} \rightleftarrows \mathrm{CoCl}_{2}+2 \mathrm{HF}$.

\begin{tabular}{c|c|c|c|c}
\hline \multirow{2}{*}{$T$} & $\mathrm{~cm}^{3} \mathrm{HCl}$ & $\mathrm{cm}^{3} \mathrm{HF}$ & $\mathrm{cm}^{3} / \mathrm{Min}$ & $\frac{H F}{H C l}$ \\
\hline \multirow{3}{*}{588} & 99.17 & 16.29 & 5.49 & 0.16 \\
& 90.23 & 22.15 & 3.51 & 0.25 \\
& 87.75 & 29.04 & 1.69 & 0.33 \\
& - & - & 0 & 0.40 \\
\multirow{3}{*}{692} & 60.39 & 24.76 & 6.55 & 0.41 \\
& 72.44 & 34.04 & $3 \cdot 60$ & 0.47 \\
& 66.24 & 33.79 & 1.84 & 0.51 \\
& - & - & 0 & 0.55 \\
& 51.73 & 36.56 & 7.23 & 0.61 \\
& 64.47 & 41.91 & 4.86 & 0.65 \\
& 57.25 & 40.07 & 1.82 & 0.70 \\
& - & - & 0 & 0.73
\end{tabular}

Das verwendete rosafarbige Salz war ein Kahlbaumsches Präparat. Es wurde wie oben angegeben getrocknet und analysiert. Die Analyse ergab $61 \cdot 2 \% \mathrm{Co}$ als Sulfat bestimmt, die Formel verlangt $60 \cdot 8 \% \mathrm{Co}$. Das entstandene $\mathrm{CoCl}_{2}$ war blassblau gefärbt.

Tabelle 17. $\mathrm{CdF}_{2}+2 \mathrm{HCl} \rightleftarrows \mathrm{CdCl}_{2}+2 \mathrm{HF}$.

\begin{tabular}{|c|c|c|c|c|}
\hline$T$ & $\mathrm{~cm}^{3} \mathrm{HCl}$ & $\mathrm{cm}^{3} H F$ & $\mathrm{~cm}^{3} / \mathrm{Min}$ & $\frac{H F}{H C l}$ \\
\hline 584 & $\begin{array}{c}90.55 \\
63.43 \\
71.28 \\
-\end{array}$ & $\begin{array}{c}25 \cdot 35 \\
23 \cdot 47 \\
32.07 \\
-\end{array}$ & $\begin{array}{l}8.88 \\
5.07 \\
1.55 \\
0\end{array}$ & $\begin{array}{l}0.28 \\
0 \cdot 37 \\
0.45 \\
0.48\end{array}$ \\
\hline 693 & $\begin{array}{c}54.14 \\
61 \cdot 51 \\
59 \cdot 33 \\
-\end{array}$ & $\begin{array}{c}59.55 \\
74.44 \\
78.93 \\
-\end{array}$ & $\begin{array}{l}7 \cdot 83 \\
5 \cdot 01 \\
1 \cdot 83 \\
0\end{array}$ & $\begin{array}{l}1.10 \\
1.21 \\
1.33 \\
1.40\end{array}$ \\
\hline 786 & $\begin{array}{c}40 \cdot 24 \\
71 \cdot 52 \\
52.48 \\
-\end{array}$ & $\begin{array}{r}75 \cdot 65 \\
163 \cdot 00 \\
130 \cdot 21 \\
-\end{array}$ & $\begin{array}{l}9 \cdot 11 \\
4 \cdot 00 \\
1 \cdot 57 \\
0\end{array}$ & $\begin{array}{l}1 \cdot 88 \\
2 \cdot 28 \\
2 \cdot 48 \\
2 \cdot 61\end{array}$ \\
\hline
\end{tabular}

Das verwendete weisse $C d F_{2}$ war ein Kahlbaumsches Präparat. Es wurde wie oben angegeben getrocknet und analysiert. Die Analyse ergab $74.4 \% \mathrm{Cd}$ als $\mathrm{CdSO}_{4}$ bestimmt, während die Formel $74 \cdot 7 \% \mathrm{Cd}$ verlangt. Das entstandene $\mathrm{CdCl}_{2}$ war weiss gefärbt. 
Tabelle 18. $\mathrm{ZnF}_{2}+2 \mathrm{HCl} \rightleftarrows \mathrm{ZnCl}_{2}+2 \mathrm{HF}$.

\begin{tabular}{|c|c|c|c|c|}
\hline$T$ & $\mathrm{~cm}^{3} \mathrm{HCl}$ & $\mathrm{cm}^{3} H F$ & $\mathrm{em}^{3} / \mathrm{Min}$ & $\frac{H F}{H C l}$ \\
\hline 590 & $\begin{array}{r}68.55 \\
181.79 \\
75.91 \\
-\end{array}$ & $\begin{array}{c}12.28 \\
48 \cdot 07 \\
29 \cdot 64 \\
-\end{array}$ & $\begin{array}{l}7 \cdot 35 \\
5 \cdot 10 \\
1 \cdot 28 \\
0\end{array}$ & $\begin{array}{l}0.18 \\
0.26 \\
0.39 \\
0.44\end{array}$ \\
\hline 682 & $\begin{array}{r}119.95 \\
77.91 \\
71.05 \\
-\end{array}$ & $\begin{array}{c}38 \cdot 45 \\
35 \cdot 84 \\
43 \cdot 34 \\
-\end{array}$ & $\begin{array}{l}7.92 \\
4.77 \\
1.71 \\
0\end{array}$ & $\begin{array}{l}0.32 \\
0.46 \\
0.61 \\
0.69\end{array}$ \\
\hline 787 & $\begin{array}{c}68 \cdot 07 \\
52 \cdot 41 \\
61 \cdot 24 \\
-\end{array}$ & $\begin{array}{c}60 \cdot 54 \\
52 \cdot 93 \\
69.21 \\
-\end{array}$ & $\begin{array}{l}8.09 \\
5 \cdot 03 \\
1.84 \\
0\end{array}$ & $\begin{array}{l}0.89 \\
1.01 \\
1.13 \\
1.19\end{array}$ \\
\hline
\end{tabular}

Das verwendete weisse $Z n F_{2}$ war ein Kahlbaumsches Präparat. Es wurde wie oben angegeben getrocknet und analysiert. Die Analyse ergab $63.7 \% \mathrm{Zn}$ als $\mathrm{Zn}\left(\mathrm{NH}_{4}\right) \mathrm{PO}_{4}$ bestimmt, die Formel verlangt $63 \cdot 4 \% \mathrm{Zn}$. Das entstandene $\mathrm{ZnCl}_{2}$ war weiss gefärbt und bei den zwei höheren Temperaturen geschmolzen.

Tabelle 19. $\mathrm{MnF}_{2}+2 \mathrm{HCl} \rightleftarrows \mathrm{MnCl}_{2}+2 \mathrm{HF}$.

\begin{tabular}{|c|c|c|c|c|}
\hline$T$ & $\mathrm{~cm}^{3} \mathrm{HCl}$ & $\mathrm{cm}^{3} H F$ & $\mathrm{~cm}^{3} / \mathrm{Min}$. & $\frac{H F}{H C l}$ \\
\hline 588 & $\begin{array}{c}75 \cdot 52 \\
77 \cdot 89 \\
47 \cdot 39 \\
-\end{array}$ & $\begin{array}{c}11 \cdot 26 \\
20 \cdot 43 \\
15 \cdot 16 \\
-\end{array}$ & $\begin{array}{l}6.67 \\
2.80 \\
0.83 \\
0\end{array}$ & $\begin{array}{l}0.15 \\
0.26 \\
0.32 \\
0.34\end{array}$ \\
\hline 673 & $\begin{array}{c}98 \cdot 47 \\
89.31 \\
75 \cdot 70 \\
-\end{array}$ & $\begin{array}{c}30 \cdot 05 \\
36 \cdot 62 \\
34.06 \\
-\end{array}$ & $\begin{array}{l}8.56 \\
4.46 \\
2.47 \\
0\end{array}$ & $\begin{array}{l}0.31 \\
0.41 \\
0.45 \\
0.51\end{array}$ \\
\hline 786 & $\begin{array}{c}79 \cdot 44 \\
52 \cdot 01 \\
45 \cdot 19 \\
-\end{array}$ & $\begin{array}{c}43,69 \\
30.69 \\
28.93 \\
-\end{array}$ & $\begin{array}{l}7.94 \\
5.00 \\
1.66 \\
0\end{array}$ & $\begin{array}{l}0.55 \\
0.59 \\
0.64 \\
0.66\end{array}$ \\
\hline
\end{tabular}

Das verwendete weisse $M n F_{2}$ war ein Kahlbaumsches Präparat. Es wurde wie oben angegeben getrocknet und analysiert. Die Analyse ergab 58.5\% $\mathrm{Mn}$ als $\mathrm{MnSO}_{4}$ bestimmt, die Formel verlangt $59 \cdot 1 \% \mathrm{Mn}$. Das entstandene $\mathrm{MnCl}_{2}$ war rosenrot gefärbt. 
Es war weiterhin beabsichtigt, das Gleichgewicht

$$
\mathrm{PbF}_{2}+2 \mathrm{HCl} \rightleftarrows \mathrm{PbCl}_{2}+2 \mathrm{HF}
$$

zu messen. Es stellte sich aber heraus, dass überhaupt kein $H F$ entwickelt wurde; es traten andere Reaktionen (Bildung von Fluorchloriden?) auf. Das Salz wurde nicht analysiert.

Die Messung des Gleichgewichts

$$
\mathrm{BaF}_{2}+2 \mathrm{HCl} \rightleftarrows \mathrm{BaCl}_{2}+2 \mathrm{HF}
$$

musste ebenfalls aufgegeben werden. Die Reaktion kam sehr bald zum Stillstand. Das entstandene $\mathrm{BaCl}_{2}$ bildete über dem $\mathrm{BaF}_{2}$ eine feste Kruste und verhinderte dann den Zutritt des $\mathrm{HCl}$ zum $\mathrm{BaF}_{2}$.

Auch auf die Messung des Gleichgewichts

$$
\mathrm{CaF}_{2}+2 \mathrm{HCl} \rightleftarrows \mathrm{CaCl} l_{2}+2 \mathrm{HF}
$$

musste verzichtet werden. Die Reaktion setzt erst bei etwa $1000^{\circ} \mathrm{C}$ ein. Eine so hohe Temperatur durften wir unserem silbernen Reaktionsrohr naturgemäss nicht zumuten.

Die Erscheinung der Oberflächenverkrustung wurde auch bei der Messung des Gleichgewichts

$$
\mathrm{NaF}+\mathrm{HCl} \rightleftarrows \mathrm{NaCl}+\mathrm{HF}
$$

beobachtet, ganz gleichgültig, ob man von reinem $N a F$ oder von einer Mischung von 50 Molproz. $\mathrm{NaF}$ mit 50 Molproz. $\mathrm{NaCl}$ ausging.

$\mathrm{Um}$ in diesem Falle zu einem ungefähren Ergebnis zu gelangen, wurde derart verfahren, dass man bei kleiner gleichbleibender Strömungsgeschwindigkeit zwei unmittelbar aufeinander folgende Messungen vornahm, um so ein Urteil über die Abnahme des Verhältnisses $\frac{H F}{H C l}$ mit der Zeit infolge der Oberflächenverkrustung zu bekommen.

Tabelle 20. NaF+HCl $\left.\rightleftarrows \mathrm{NaCl}+H F^{1}\right)$.

\begin{tabular}{c|c|c|c|c}
\hline$T$ & $\mathrm{em}^{3} \mathrm{HCl}$ & $\mathrm{cm}^{3} \mathrm{HF}$ & $\mathrm{em}^{3} / \mathrm{Min}$. & $\frac{H F}{H C i}$ \\
\hline \multirow{2}{*}{563} & 23.04 & 88.59 & 1.6 & $3 \cdot 8$ \\
& 26.17 & 88.03 & $1 \cdot 6$ & $3 \cdot 4$ \\
\multirow{2}{*}{624} & 1.23 & 124.94 & $2 \cdot 1$ & 101.5 \\
& 3.17 & 156.33 & $2 \cdot 6$ & $49 \cdot 3$
\end{tabular}

1) Als Analogon zu diesen Messungen vgl. die Arbeit von JeLLinek und ITELSOHN, loc. cit. 
Das verwendete weisse $N a F$ war ein Kahlbaumsches Präparat pro analysi. Das Mischungsverhältnis der festen Phase hatte keinen

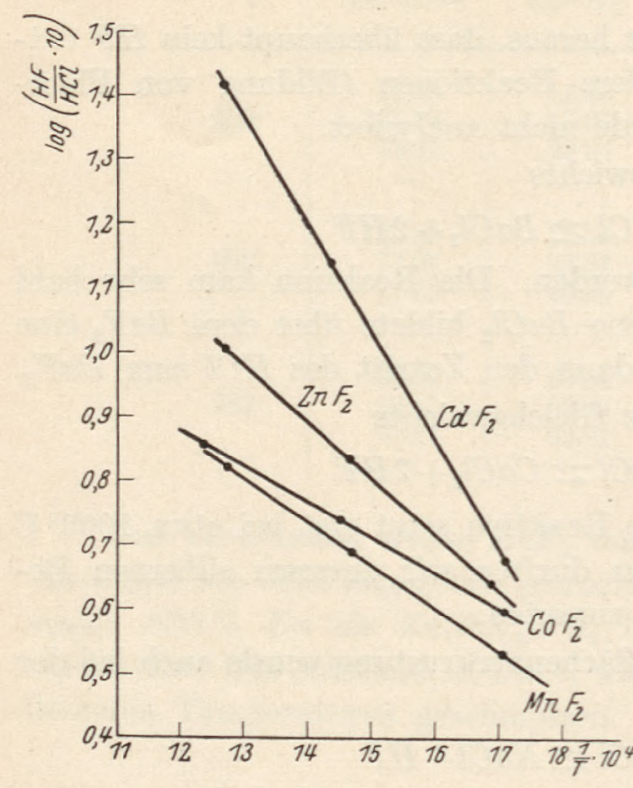

Fig. 9 . Einfluss auf das Resultat. Das Gleichgewichtliegt bei $T=624^{\circ}$ schon jedenfalls sehr stark nach der $H F$-Seite hin verschoben. In Fig. 9 sind die Gleichgewichtefür $C d F_{2}, Z n F_{2}$, $\mathrm{CoF}_{2}$ und $\mathrm{MnF}_{2}$ graphisch zusammengefasst. Trägt man für jedes Metallfluorid $\log K=\log$ $\frac{H F}{H C l}$ gegen $\frac{1}{T}$ auf, so ergibt die Kurve eine gerade Linie (Fig. 9).

\section{Berechnung der Wärmetönung} der Reaktionen

$\mathrm{MeF}_{2}+2 \mathrm{HCl} \rightleftarrows \mathrm{MeCl}_{2}+2 \mathrm{HF}$.

Die Berechnung geschieht nach der Formel $\log K_{1}-\log K_{2}=\frac{Q}{4 \cdot 57}\left(\frac{1}{T_{1}}-\frac{1}{T_{2}}\right)$, wo $K_{1}$ und $K_{2}$ die Gleichgewichtskonstanten bei der Temperatur $T_{1}$ und $T_{2}$ bedeuten. Da wir für $K$ den Wert $\frac{H F}{H C l}$ in die Formel einsetzen, finden wir die Wärmetönung pro $1 \mathrm{Mol}$ entstehendes $H F$.

In Tabelle 21 sind für jedes Metallfluorid eingetragen: Die Temperatur $T$, ferner $\log K=\log \frac{H F}{H C l}$ und die aus obiger Formel berechnete, mit 2 multiplizierte Wärmetönung $Q$ in Calorien.

Tabelle 21.

\begin{tabular}{c|c|c|c}
\hline Reaktion & $T$ & \multicolumn{1}{c}{$\log K$} & $Q$ in cal \\
\hline $\mathrm{CoF}_{2}+2 \mathrm{HCl}=\mathrm{CoCl}_{2}+2 \mathrm{HF}$ & 588 & $0.6021-1$ & -5190 \\
$\mathrm{CdF}_{2}+2 \mathrm{HCl}=\mathrm{CdCl}_{2}+2 \mathrm{HF}$ & 805 & $0.8633-1$ & - \\
& 584 & $0.6812-1$ & -15274 \\
$\mathrm{ZnF}_{2}+2 \mathrm{HCl}=\mathrm{ZnCl}_{2}+2 \mathrm{HF}$ & 586 & 0.4166 & \\
& 590 & $0.6435-1$ & -9402 \\
$\mathrm{MnF}_{2}+2 \mathrm{HCl}=\mathrm{MnCl}_{2}+2 \mathrm{HF}$. & 587 & 0.0755 & \\
& 786 & $0.5315-1$ & -6122
\end{tabular}


Die Wärmetönungen ergeben sich also in allen Fällen negativ und sind relativ klein. Zu einer genauen Kontrolle dieser Zahlen reichen unsere Kenntnisse der Bildungswärme von $\mathrm{HF}, \mathrm{HCl}$, der Metallfluoride und Metallchloride, bzw. ihrer Temperaturkoeffizienten nicht aus. Aus diesen Bildungswärmen würden sich unsere Wärmetönungen als sehr unsichere Differenzen grosser Zahlen ergeben. Jedenfalls aber ist durch die vorausgehenden Messungen der Gleichgewichte sichergestellt, dass der Fluorwasserstoff aus den untersuchten Fluoriden leicht durch $\mathrm{HCl}$ vertrieben werden kann.

\section{Zusammenfassung.}

1. Es werden die Reduktionsgleichgewichte folgender Metallhalogenide mit Wasserstoff gemessen, wobei die Temperaturen zwischen $200^{\circ}$ und $1100^{\circ} \mathrm{C}$ lagen: $\mathrm{CoF}_{3}$ zu $\mathrm{CoF}_{2}, \mathrm{MnF}_{2}, \mathrm{FeCl}_{3}$ zu $\mathrm{FeCl}, \mathrm{CrCl}_{3}$ zu $\mathrm{CrCl}_{2}, \mathrm{CrCl}_{2}, \mathrm{CuCl}_{2}$ zu $\mathrm{Cu}_{2} \mathrm{Cl}_{2}$.

2. Die Halogentensionskurven der Metallfluoride und Chloride werden berechnet und die Bildungswärmen der Metallhalogenide aus den Halogentensionen ermittelt. Die Übereinstimmung mit den calorimetrisch gemessenen Wärmetönungen ist im allgemeinen befriedigend.

3 . Es werden die schwierig festzustellenden Gleichgewichte folgender Metallfluoride mit Chlorwasserstoff zwischen $300^{\circ}$ und $500^{\circ} \mathrm{C}$ gemessen: $\mathrm{CoF}_{2}, \mathrm{CdF}_{2}, \mathrm{ZnF}_{2}, \mathrm{MnF}_{2}, \mathrm{NaF}$.

4. Die Wärmetönungen der Reaktionen unter 3. werden aus den Gleichgewichtskonstanten ermittelt.

5. Die Dampfspannungen von $\mathrm{FeCl}_{3}, \mathrm{CrCl}_{3}$ und $\mathrm{ZnCl}_{2}$ werden zwischen $200^{\circ}$ und $280^{\circ} \mathrm{C}$, bzw. zwischen $700^{\circ}$ und $1000^{\circ} \mathrm{C}$, bzw. zwischen $600^{\circ}$ und $700^{\circ} \mathrm{C}$ ermittelt.

Bei unserer Untersuchung standen uns Mittel der Notgemeinschaft der Deutschen Wissenschaft zur Verfügung, wofür bestens gedankt sei.

Danzig, Analyt. Abt. d. Anorgan.-Chem. Instituts d. Techn. Hochschule.

September 1929. 


\section{Lebenslauf.}

Ich, Ernst Reinhard Erhard Koop, wurde am 18. September 1901 als Sohn des Molkereibetriebsleiters Ernst August Koop in Winzig, Kreis Wohlau, geboren. Von Ostern 1907 bis Ostern 1912 besuchte ich in Wohlau die Volksund Privatschule. Von Ostern 1912 bis Ostern 1918 war ich Schüler des Humanistischen Gymnasiums in Wohlau. Seit Ostern 1918 besuchte ich das Realgymnasium zu Jüterbog und legte dort Ostern 1922 die Reifeprüfung ab. Im Mai 1922 begann ich an der Universität in München ein Medizinstudium. Nach drei Semestern immatrikulierte ich mich an der Technischen Hochschule in München als Studierender der Chemie und legte im Juli 1926 das Vorexamen für Chemie ab. Seit Mai 1927 bin ich an der Technischen Hochschule in Danzig eingeschrieben. Hier bestand ich im Juli 1928 das Diplomhauptexamen für Chemie. Die vorliegende Arbeit wurde in der Zeit vom 15. Oktober 1928 bis zum 15. September 1929 ausgeführt. Am 2. November 1929 bestand ich die mündliche Doktorprüfung. Für die Anregung zu vorliegender Arbeit und für die Ratschläge bei ihrer Durchführung danke ich meinem hochverehrten Lehrer, Herrn Prof. Dr. K. Jellinek, aufs wärmste.

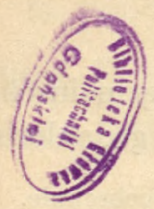




\section{BIBLIOTEKA GtOWNA}

II 38604

Politechniki Gdanskiej 